\title{
Das Lößprofil von Leitmeritz an der Elbe, Nordböhmen
}

\author{
Von Vojen Ložek und Jiří Kukla, Prag \\ Mit einer Tafel (I) und 3 Tabellen
}

$\mathrm{Z}$ us a mmen $\mathrm{f}$ as su ng. In der Ziegelei von Leitmeritz (= Litoměřice) (Nordböhmen) ist eine reich gegliederte Schichtenfolge jungpleistozäner Lösse und fossiler Humusböden aufgeschlossen, die zeitlich der Würmeiszeit bzw. dem letzten Interglazial und dem Göttweiger Interstadial entspricht. Das Profil wurde eingehend stratigraphisch und petrographisch untersucht. In zwei Humushorizonten des erwähnten Interstadials wurde eine reiche Molluskenfauna festgestellt, die größtenteils aus wenig temperaturanspruchsvollen Steppenarten besteht und sich sowohl von der lezztinterglazialen Fauna als auch von den Lößgesellschaften stark unterscheidet. Aus der Analyse der untersuchten Schneckenfaunen ergibt sich, daß das Klima des Göttweiger Interstadials verhältnismäßig trocken und winterkalt war. Denselben Charakter weisen auch die Molluskenfaunen auf, die im Göttweiger Interstadial an anderen tschechoslowakischen Fundstellen festgestellt wur$\mathrm{d} e \mathrm{n}$. Aus dem gegenseitigen Vergleich verschiedener Lößprofile in der Tschechoslowakei, Oisterreich und Süddeutschland ergibt sich die Möglichkeit, daß im fossilen Bodenkomplex, der als Stillfried A bezeichnet wird, nicht nur das Letzte Interglazial sondern auch das Göttweiger Interstadial verborgen sind. Wenn es gelingen würde, diese Voraussetzung durch verläßliche Beweise zu bestätigen, wäre der Hauptwiderspruch in der stratigraphischen Untergliederung der würmeiszeitlishen Lößprofile gelöst. Aus vergleichenden Untersuchungen der letztinterglazialen und würminterstadialen Molluskenfauna ist ersichtlich, daß man das Interglazial als Schwankung einer höheren Ordnung vom Interstadial scharf unterscheiden muß, wobei man aber in Betracht ziehen sollte, daß das Göttweiger Interstadial wieder eine Schwankung höherer Ordnung ist als die anderen Interstadiale der Würmeiszeit, für welche in Übereinstimmung mit dem Vorschlag von H. Movius (1957) der Termin „Oszillation“ geeignet wäre.

A bstract. In the brickyard at Litoměřice (Northern Bohemia) there is a complicated outcrop in a complex of loesses and fossil humic soils which probably correspond to the Last Interglacial and to the Göttweig Interstadial. In three humic horizons of this Interstadial a rich mollusc fauna has been found. This fauna consists mostly of climatically tolerant steppe species and differs sharply from the mollusc communities of the Last Interglacial and from the loess fauna too. The analysis of these mollusc finds gives evidence that the climate of the Göttweig Interstadial was relatively dry with long cold winters. The mollusc faunae of the Göttweig Interstadial in other Czechoslovak localities are of the same type. The critical comparison of different loess sections in Czechoslovakia, Austria and Southern Germany suggests that the fossil soil complex named Stillfried A probably includes both the Last Interglacial and the Göttweig Interstadial. If this hypothesis were surely verified, the main controversy in the stratigraphy of the Wirmian loess sectiors would be solved. From the comparative studies of the molluscs of the Last Interglacial and of the Würmian Interstadials results the necessity to distinguish the Interglacial as a climatic change of higher rank from the Interstadials. It must be emphasized that also the Göttweig Interstadial is a change of higher rank than the other warm periods of the Würmian for which it would be most convenient to use the term "oscillation" in agreement with the proposal of $\mathrm{H}$. Movius (1957).

\section{Einleitung}

In den letzten Jahren wurde der stratigraphischen Feingliederung des Jungpleistozäns, besonders der Würmeiszeit, viel Aufmerksamkeit gewidmet (vgl. Woldstedt 1956). Eine sehr wichtige, man könnte sagen die Hauptrolle, spielten und spielen hier die Lößprofile in warmtrockenen Gebieten Mitteleuropas. Die größte Zahl der jungpleistozänen Lößkomplexe wurde in Niederösterreich durchforscht, wo auch das stratigraphische Grundsystem ausgearbeitet wurde (GöTzINGER 1935; BrANDTNER 1954, 1956; FinK 1954, 1956). Weitere bedeutende Gebiete sind Innerböhmen, Süiddeutschland, Südmähren und die Südwestslowakei.

Heutzutage gibt es zwei Hauptansichten über die stratigraphische Gliederung der würmeiszeitlichen Lößkomplexe. Einerseits ist es die Auffassung, daß das Würm in drei Hauptstadiale zerfällt, die voneinander durch zwei Interstadiale getrennt sind, von 
denen das ältere viel ausgeprägter ist als das jüngere. Diese Ansicht ist in verschiedenen lokalen Abänderungen in der Tschechoslowakei anerkannt (vgl. ProšEK \& LožEK 1957) und wird auch von einigen deutschen (Gross 1956, 1958; Woldstedt 1956, 1958) und österreichischen Autoren (BrandTNER 1950, 1954, 1956) vertreten. Woldstedt (1958) übernahm diese Gliederung auch in sein zusammenfassendes Werk über das Quartär, wo er für die ältere warme Schwankung den Namen "Göttweiger Interstadial“ in Übereinstimmung mit H. GRoss (1956), für die jüngere schwächere Schwankung die Bezeichnung „Paudorfer Interstadia ${ }^{\text {“ }}$ wählt. Diese Namen sind von klassischen österreichischen Fundorten abgeleitet. Man muß hier jedoch hervorheben, daß die Göttweiger Schwankung, welche in der tschechoslowakischen Literatur gewöhnlich nur mit dem Index „W 1/2" bezeichnet wird, in den Profilen durch einen komplizierten fossilen Bodenkomplex dargestellt wird, welchem BrandTNer (1954) die Bezeichnung „F e 11 abrunner Komplex" gibt, während Fink (1954) ihn als "Stillf rieder Komplex A“, bzw. als "Linzer Komplex" in der Feuchtlandschaft bezeichnet. Demgegenüber entspricht der Paudorfer Schwankung nur ein schwach humoser Boden im Trockengebiet („Stillfried B“ bei $F_{1 N K} 1954$ ), bzw. der sogenannte $\mathrm{N}$ a ß boden in der Feuchtlandschaft.

Andererseits gibt es die zweite Auffassung, welche vor allem von süddeutschen und österreichischen Geologen (BrunNaCker 1955, 1957, 1958; Freising 1951, 1956; GötZiNGer 1935; Vetters \& Götzinger 1936; WeIdenbach 1952, 1956 und in gewissem Maße auch Fink 1954, 1956 und MüLler-BeCK 1957) vertreten wird, die voraussetzt, daß das Würm der Lößkomplexe nur zweiteilig ist, d. h. daß der entsprechende Lößkomplex nur durch eine schwache Schwankung getrennt ist, welche dem Paudorfer Interstadial entspricht. Demgegenüber wird der Bodenkomplex des Göttweiger Interstadials, bzw. seine basale Verlehmungszone, von diesen Autoren bereits mit dem Letzten Interglazial (R/W, Eem) identifiziert. Diese Problematik wurde neuestens von K. Brunnacker (1958) ausführlich zusammengefaßt.

Den Widerspruch zwischen den beiden Ansichten kann man kurz in dem Sinne zusammenfassen, daß beide Gruppen der Autoren in ihrer Auffassung der Position des letzten echten Interglazials in den Lößprofilen a usein a ndergehen. Die eine Gruppe identifiziert es mit dem fossilen Bodenkomplex im Liegenden des "Jüngeren Lösses I“ (=Altwürm-Löß, W 1-Löß der tschechoslowakischen Autoren), auf dem der Stillfrieder Komplex A ruht, die anderen halten bereits den Stillfrieder Komplex A für letztinterglazial.

Die paläontologischen Daten, welche eine von diesen Ansichten unterstützen würden, sind bis jetzt ungenügend (vgl. Gross 1958, bes. S. 168), und in der Literatur ist noch nicht einmal der Unterschied zwischen der Fauna des echten Interglazials und des Interstadials gründlich festgestellt, abgesehen von einigen allgemeinen oder wieder lokalen Angaben (vgl. Gross 1958; VÉRtes 1955; LožEK 1955 usw.). Vom tschechoslowakischen Gebiet ist zwar eine ganze Reihe Fundstellen der hochinterglazialen Banatica-Fauna aus den Lößprofilen bekannt, es handelt sich leider jedoch nur um solche Schichtenfolgen, welche ein wenig anders entwickelt sind als die klassischen österreichischen Profile und bisher nicht eingehend untersucht wurden, so daß eine genaue Parallelisierung unsicher bleibt. Dabei muß man hervorheben, daß die meisten von diesen tschechoslowakischen Profilen (Sedlec bei Prag, Letky, Zamarovce) für die durch Gross, Woldstedt und Brandtner vertretene Ansicht zeugen, d. h. für die Dreiteilung der Würmeiszeit (ProšEK \& LožEK 1957).

Unser Aufsatz gibt eine eingehende stratigraphische Analyse des Lößprofils von Leitmeritz an der Elbe in Nordböhmen, wo es gelungen ist, eine reiche Molluskenfauna in mehreren Humushorizonten festzustellen, welche auffallend an den Stillfrieder Komplex erinnern. Hier wollen wir ausführlich die Thanatozönosen des Leitmeritzer Profils be- 
schreiben, ihre genaue Kennzeichnung geben und auf ihre starke Verschiedenheit von den letztinterglazialen Molluskengesellschaften hinweisen. Die eingehende Bearbeitung des Leitmeritzer Profils ergänzen wir durch einige Erwägungen über die allgemeine stratigraphische Problematik der mitteleuropäischen Lößßprofile, welche einerseits zur Lösung des Widerspruches zwischen den beiden obenerwähnten Ansichten beitragen und andererseits der Klärung einiger allgemeiner Begriffe, wie z. B. Interglazial und Interstadial, dienen sollen, deren gegenseitige Unterschiede noch nicht mit genügender Genauigkeit festgestellt sind, was oft zu ernsten Mißverständnissen führt.

\section{Der Fundort und seine Umgebung}

Das untersuchte Profil befindet sich in der Nordostwand einer verlassenen Ziegelei am Ostrand der Stadt Leitmeritz (Litoměrice) an der Straße nach Böhmisch-Leipa (Česká Lípa). Es ist $80 \mathrm{~m}$ in nordwestlicher Richtung vom Rande der Straße entfernt. Die Oberkante der erwähnten Lößwand liegt in einer Seehöhe von $184 \mathrm{~m}$. Der Ort liegt in geschützter Lage im Elbtal am Südfuß des Böhmischen Mittelgebirges. Die durchschnittliche Jahrestemperatur ist $8,9^{\circ} \mathrm{C}$, die Niederschläge betragen $507 \mathrm{~mm}$. Die nähere Umgebung der Lokalität ist heute verbaut oder landwirtschaftlich ausgenützt, so daß hier die ursprïnglichen Naturverhältnisse nicht mehr erhalten sind. In der weiteren Gegend herrschen aber xerotherme Steppenbestände mit sehr reicher Fauna und Flora vor, die mit warmen Hainen abwechseln, welche von den Südhängen des Mittelgebirges herabsteigen. In der breiten Aue der Elbe, in die hier der Egerfluß einmündet, waren ursprünglich ausgedehnte Auenwälder vorhanden, die heute nur in spärlichen Resten erhalten sind. Aus diesen Angaben ist ersichtlich, daß die Umgebung von Leitmeritz zu den wärmsten Landschaften Böhmens gehört und daß sie in der nördlichen Randzone der innerböhmischen Steppenregion liegt, die pedologisch durch die Böden von gewöhnlich schwach degradiertem Tschernosem-Typus gekennzeichnet ist.

Die Lößdecke, in der die Lehmgrube der Ziegelei aufgeschlossen ist, befindet sich im sanft welligen Gelände am Westhang eines flachen Rückens, der vom Norden zum Elbtal herabläuft. Das Elbtal breitet sich hier in ein geräumiges Talbecken aus, an dessen Nordrand Leitmeritz liegt. Im erwähnten 'Talbecken und an seinen Rändern befindet sich ein reich gegliedertes System der Elbe- und Egerterrassen, die aber durchaus am linken Elbeufer liegen, während das rechte Ufer, wo sich auch unser Profil befindet, nur kleinere größtenteils lößbedeckte Terrassenrelikte trägt. Deshalb muß man bei der Korrelation des untersuchten Lößkomplexes und der Elbeterrassen vor allem die genau festgestellte Seehöhe der Profilsohle in Betracht ziehen, aus der das Höhenverhältnis des Lößpaketes zu den einzelnen Terrassenstufen ersichtlich ist. Die Seehöhe des Elbspiegels südlich vom Profil beträgt 137-138 m, die tiefste erreichte Stelle des untersuchten Profils liegt 171,5 m hoch. $\mathrm{Da}$ das Liegende der Lösse nicht erreicht wurde, kann man voraussetzen, daß die Lößschichten noch tiefer, mindestens bis $170 \mathrm{~m}$ oder noch mehr reichen. Die Profilsohle liegt also ungefähr 30-32 m über dem Flußspiegel, also im Bereiche der Oberkante der Altriß-Terrassen (Stufe IIIb nach 'ZAHÁLKA 1946). Auch wenn die genaue Korrelation mit den Terrassenstufen eingehenderer geologischer Untersuchungen bedürfen würde, kann man dennoch mit Sicherheit behaupten, daß das Profil viel jünger ist als die II. Terrassengruppe, die gewöhnlich mit der Mindeleiszeit parallelisiert wird. Der untersuchte Lößkomplex muß also jünger als das Holsteiner Interglazial sein.

Der Felsgrund in der nahen Umgebung der Ziegelei ist von oberturonen Mergeln und feinsandigen Kalkmergeln (Pläner) gebildet, in deren Hangendem weiche Mergel des Oberturons und Emschers in der weiteren Umgebung liegen. Das Böhmische Mittelgebirge besteht aus tertiären basischen Ergußgesteinen, hauptsächlich aus Basalt und Tephrit und ihren Tuffen, in deren Liegendem eine sandige Schichtenfolge liegt, die für oligozän gehalten wird. 


\section{Beschreibung des Profils}

In der gesamten Länge der untersuchten Lößwand kann man im unteren Abschnitt einige parallel verlaufende Humushorizonte beobachten. Am besten sind diese Horizonte im mittleren Teil der Lößwand aufgeschlossen, wo wir eingehende stratigraphische Untersuchungen des Lößkomplexes durchgeführt haben. Dieser Aufschluß wurde am Fuße der Wand durch eine Schurfsonde ergänzt, die $15 \mathrm{~m}$ nach Süden vom unteren Ende des beschriebenen offenen Profils entfernt ist, welches die oberen 7,5 $\mathrm{m}$ der Lößschichtenfolge erfaßt.

Eine ausführliche Beschreibung des Profils findet man in der beigefügten Tabelle ('Taf. I), die alle festgestellten Angaben ïber verschiedene Eigenschaften der einzelnen Schichten ïbersichtlich zusammenfaßt. In groben Zügen kann man im Profil folgende stratigraphische Haupteinheiten unterscheiden:

A. Holozäner, bzw. rezenter Boden

B. Obere Lösse mit einem Fließerdehorizont an der Basis

C. Grobkörniger Löß aus Mergeldetritus

D. Obere Gruppe humoser Böden

E. Komplex vorwiegend abgespülter Mergel mit einer Lage von grobkörnigem Löß

F. Untere Gruppe humoser Böden

G. Basislöß mit Fließerdeeinlagerungen

(Das Liegende der Lösse wurde nicht erreicht).

Aus allen unterscheidbaren Schichten wurden Proben zu petrographischen und paläontologischen Analysen entnommen. Mollusken wurden insgesamt in 8 Schichten festgestellt. Diese Thanatozönosen werden im weiteren Text mit den Abkürzungen MF bezeichnet, die von oben nach unten numeriert sind (MF 8 ist also die älteste Molluskenfauna).

Wie bereits erwähnt, sind die Ergebnisse der petrographischen Untersuchung in der beigelegten Übersichtstabelle (Taf. I) kurz zusammengefaßt. In Hinsicht auf die Tatsache, daß diese Arbeitsweise noch nicht in der Fachliteratur normalisiert ist und in der Tabelle Ergebnisse einiger Methoden angeführt werden, die man noch nicht üblicherweise benïtzt, müssen wir einige erklärende Worte vorausschicken.

Erläuterungen zur petrographischen Ubersichtstabelle (Taf. I). Die Tafel ist in 31 senkrechte Kolonnen geteilt, von denen die Spalten 1-5 die Hauptdaten über das Profil enthalten, und zwar die graphische Darstellung der gesamten Schichtenfolge mit eingezeichneten Stellen der Probenentnahme, mit Angaben über die Seehöhe und den Abstand der Schichten von der Oberfläche. Die Bedeutung der benützten Kennzeichen ist aus der petrographischen Beschreibung der einzelnen Schichten ersichtlich (Koll. 9-11). Es werden hier zwei Gruppen von Kennzeichen benützt. Die eine bezeichnet die Art der Sedimentation, die andere Veränderungen, die in der Schicht durch die Bodenbildung verursacht wurden. Stärker humose Bodenhorizonte sind mit einer senkrechten Wellenlinie \}, nichthumose mit einer einfachen Linie | markiert. Die Zonen der Karbonatanreicherung (Ca-Horizonte) werden durch Kreuze an diesen senkrechten Linien bezeichnet. Die Pfeile $\uparrow$ und die Zeichen S 1-S 6 bedeuten im schematischen Profil die unteren Grenzen der Lößdecken. Es muß darauf hingewiesen werden, daß im Profil die scharfen Schichtgrenzen (volle Linien) von den allmählichen Übergängen (gestrichelte Linien) unterschieden werden. In der Spalte 6 befindet sich ein sedimentologisches und pedologisches Schema des Profils, das auf Grund aller in Tafel I enthaltenen Daten zusammengestellt wurde. Die Kolonnen 7 und 8 enthalten die Indexbezeichnungen der Schichten und der Molluskenfaunen, die im weiteren Text überall angeführt werden. Die Spalten 9 und 11 sind der grundsätzlichen petrographischen Charakteristik gewidmet, wobei die Farben mit folgenden Abkürzungen bezeichnet sind: 1 - licht, d - dunkel, S - schwarz, 
B - braun, G - gelb, Gr - grau, W - weiß, Gn - grünlich, O - ockergelb. Die Abkürzungen werden so gestellt, daß die vorherrschende Schattierung zuletzt angegeben ist( GB - gelbbraun), eine schwache Schattierung ('Tönung) wird in Klammer gestellt ([GR]B - braun mit grauer Tönung). Sind die Farbenschattierungen unregelmäßig vermengt, $d$. h. bildet eine bestimmte Farbe Schlieren oder Flecke auf einem andersfarbigen Untergrund, so wird die Bezeichnung der Farbe dieser Flecke vor einen schräggestellten Strich gesetzt (G/S - schwarz mit gelben Flecken). In der 11. Spalte sind verschiedene Beimischungen in der Grundmasse der Schichten beschrieben, insofern sie von stratigraphischer Bedeutung sind.

In den Kolonnen 12-17 sind die wichtigsten Textur ${ }^{1}$-Merkmale beschrieben, d. h. die Aggregatbildung und die mittlere Aggregatgröße, weiter die Häufigkeit und durchschnittliche Größe (in mm) der Wurzelröhrchen $(14,15)$ und größerer Leitbahnen, meistens der Wurmgänge (W - Wurmgänge, R - Wurzelröhre). Die Absonderung und Aggregatbildung werden folgenderweise kurz gekennzeichnet (Sp. 12): B - blockprismatisch, P - polyederbildend, k - krümelig, F - Feinaggregate, E - verkittetes Einzelkorngefüge. Gibt es in einer Schicht zwei oder mehrere Arten der Aggregatbildung, bzw. Absonderung, so werden alle Arten angeführt, wobei die vorherrschende immer hinter einen schräggestellten Strich gesetzt wird. Ahnliche Bezeichnungen werden auch in weiteren Spalten der Tabelle benützt. Die relative Häufigkeit verschiedener Bildungen (gültig für die ganze Tabelle) wird durch folgende Skala ausgedrückt: 1 - sehr häufig, 2 - häufig, 3 - mittelhäufig, 4 - selten, 5 - nicht vorhanden.

Diese Daten wurden teils durch direkte Becbachtung im Gelände, teils durch Untersuchung mit dem Binokular erhalten.

Die Struktur ${ }^{1}$ )-Eigenschaften sind in den Kolonnen 18-22. zusammengefaßt. Die Abkürzungen in der Spalte 18 haben folgende Bedeutung: L - pelitisch, A - aleuritisch, P psammitisch; o- homogen (nicht orientiert), $X$ - nicht homogen (nicht orientiert), = - geschichtet.

In der Spalte 20 sind die Kleinporen durch folgende Zeichen beschrieben: L - regelmäßige häufige Feinporen (tvpisch für Löß), E - einzelne Poren, G - grobe Poren. Die relative Häufigkeit der Poren ist durch die bereits erwähnte Skala (1-5) angegeben. Sekundäre Ausscheidungen von Karbonat (Kol. 21) und Sesquioxyden (Kol. 22): F - rostfarbiger Limonit, M - schwärzlicher Manganolimonit. Die Ausscheidungen in den Leitbahnen (Wurmgänge und Wurzelröhrchen) sind mit dem Index 1 bezeichnet (in der Grundmasse ohne Bezeichnung). Die Strukturmerkmale wurden mit Hilfe der Dünnschliffe und der Beobachtung unter dem Binokular bestimmt.

Die Spalten 23-26 enthalten die wichtigsten Daten der Kornanalyse, die bei allen Proben mit der Casagrande-Methode durchgeführt wurde (es handelt sich um folgende Proben: L 1-22, 201-204, 212, 214, 90, 94). In der Kolonne 25 ist das Verhältnis der $\%$ II. Kategorie

I. und II. Kategorie nach der Formel _-___- 100 angegeben. Diese Kennzahl $\%$ I. Kategorie

ist beim Studium der Lösse von größter Bedeutung, besonders in Gebieten, wo die Lösse einen höheren Anteil von leicht zersetzbaren Mineralkörnern nicht enthalten. In einer sekundär nicht veränderten Lößdecke steigt die erwähnte Kennzahl gesetzmäßig vom Liegenden zum Hangenden. Im Falle von Leitmeritz konnte man diese Regel stratigraphisch nicht völlig ausnützen, da die Leitmeritzer Lösse einen hohen Anteil von Mergeldetritus enthalten, der sich im Verlaufe der Bodenbildung zersetzt, so daß die ursprüngliche Körnung nicht erhalten bleibt.

1) Die Begriffe Textur und Struktur werden hier stets in der petrographischen, d. h. nicht in der pedologischen Bedeutung benützt. 
Ein anderes wichtiges Kennzeichen ist der sogenannte Wendepunkt der Körnungskurve (Spalte 26). Dieser Punkt bezeichnet die maximale Größe der Partikel, die in der sedimentierten Schicht noch in beträchtlicher Menge abgelagert wurden. Er wird folgenderweise bestimmt: man verbindet je zwei nächste Fixpunkte (die während der Casagrande-Analyse festgestellt wurden) beiderseits der oberen Biegung der Körnungskurve durch gerade Linien, deren Schnittpunkt den gesuchten Wendepunkt bestimmt. Wenn die Biegung der Kurve nicht genügend ausgeprägt ist, wird die Stellung des Wendepunktes undeutlich, und die erhaltenen Werte werden in Klammern angegeben; ein besonders ausgeprägter Wendepunkt ist unterstrichen. In einer Lößablagerung, die sich während einer Sedimentationsphase gebildet hat, sinkt der Anteil der gröberen Körner von unten nach oben gesetzmäßig herab, was sich in der Stellung des Wendepunktes deutlich bemerkbar macht. Auf Grund dieser Beobachtung kann man in unserem Profil mindestens fünf selbständige Phasen der Lößablagerung unterscheiden, welche an ihrer Basis grobes Material führen, das aus über 0,1 mm großen Körnern besteht.

In der Kolonne 27 ist der Karbonatgehalt in den Körnungskategorien unter 0,2 mm durch eine Kurve dargestellt, deren Fixpunkte mit der Probenummer und dem genauen Wert des Karbonatgehaltes bezeichnet sind (analoge Bezeichnungen gibt es auch in den Kolonnen 28 und 30). Die Spalte 28 enthält eine Kurve des Volumengewichtes und Angaben über den relativen Humusgehalt einiger Schichten. Das Volumengewicht wird auf zweierlei Weise festgestellt; im Falle, daß die untersuchten Erden feucht sind, benützt man einen Metallzylinder für ungestörte Probenentnahme; die Probe wird bei $110^{\circ} \mathrm{C}$ ausgetrocknet und gewogen; aus dem bekannten Volumen des Zylinders kann man dann die gesuchte Zahl ausrechnen. Im Falle, daß die Erden trocken sind, was die obenbeschriebene Probenentnahme verhindert, muß man größere ungestörte Stücke aus den Schichten herausheben. Solche Proben werden im Laboratorium wieder bei $110^{\circ} \mathrm{C}$ ausgetrocknet; ihr Volumen wird dann in einem mit viskoser Flüssigkeit (z. B. Glyzerin) gefüllten Meßzylinder festgestellt. Aus dem Verlaufe der Kurve ergibt sich, daß das Volumengewicht beim primären Löß in Leitmeritz zwischen 1,5 - 1,6 g/ $\mathrm{cm}^{3}$ schwankt. Bei den dichten abgeschwemmten Ablagerungen beträgt es $1,7-1,8 \mathrm{~g} / \mathrm{cm}^{3}$, bei autochthonen Böden ist es am niedrigsten $-1,4-1,5 \mathrm{~g} / \mathrm{cm}^{3}$. Mit Hilfe des Volumengewichtes kann man autochthone Böden von umgelagerten Bodensedimenten mit Sicherheit unterscheiden (vgl. die Proben 87 und 85 ).

Der relative Humusgehalt wurde kolorimetrisch im Vergleich mit dem postglazialen Boden festgestellt (vgl. LaIs, 1958). Die Nummer der untersuchten Proben und der festgestellte Humusgehalt in \% ergänzen die graphische Darstellung. Man muß hier in Betracht ziehen, daß die Grundprobe $(=100 \%)$ einem Ackerboden entnommen wurde, der durch die Tätigkeit des Menschen sekundär um organische Stoffe bereichert ist, so daß man voraussetzen kann, daß sein ursprïnglicher Humusgehalt niedriger war.

Den relativen Glimmergehalt (Kol. 29) in der Kategorie 0,02-0,1 mm (Fixpunkte mit Kreisen markiert) und $0,1-0,2 \mathrm{~mm}$ (Fixpunkte mit Kreuzen bezeichnet; Häufigkeit s. Kol. 14) kann man auf Grund der Tatsache stratigraphisch ausnützen, daß die Glimmerblättchen bei gleichem Gewicht eine viel größere Oberfläche als andere Mineralteilchen besitzen und deswegen viel leichter vom Winde transportiert werden können. Deshalb ist der Glimmergehalt der Lösse höher als in den Ausgangsgesteinen, von denen das Lößmaterial ausgeweht wurde. Im untersuchten Profil haben die oberen und unteren Lößschichten einen erhöhten Glimmergehalt, der größtenteils aus den Elbeterrassen stammt und vorwiegend aus baueritisiertem Biotit besteht. Dagegen ist im Bodenkomplex der Glimmeranteil niedriger. Das Ansteigen des Glimmeranteils in der Probe L 19 kennzeichnet den Anfang einer neuen Lößsedimentation.

In der Kolonne 30 ist der Prozentgehalt der Karbonatkörner (Kalkstein, Pläner, Mergel usw.) in der Fraktion 0,1-0,2 mm angegeben. Das Abnehmen des Karbonatgehaltes 
in dieser Fraktion kennzeichnet die bodenbildenden Prozesse. Die planimetrisch genau festgestellten Daten sind in der Tabelle unterstrichen, die übrigen wurden schätzungsweise erhalten. Eine stärkere chemische Korrosion der Karbonatkörner ist durch kleine Pfeile bezeichnet.

\section{Die Molluskenfauna}

Die Schneckenfunde sind in zwei Tabellen zusammengefaßt, von denen die eine die wahre Zahl der gesammelten Individuen angibt, die andere alle bisher bekannten tschechoslowakischen Schneckenfunde aus dem Göttweiger Interstadial zusammenfaßt.

I. Tabelle der Molluskenfunde

\begin{tabular}{|c|c|c|c|c|c|c|c|c|}
\hline \multirow[b]{2}{*}{ Arten } & \multicolumn{8}{|c|}{ Thanatozönosen } \\
\hline & MF 8 & MF 7 & MF 6 & MF 5 & MF 4 & MF 3 & MF 2 & MF 1 \\
\hline Succinea sp. & - & - & - & - & - & 4 & 1 & - \\
\hline Cocblicopa sp. & -- & 2 & - & - & - & - & - & - \\
\hline Abida frumentum (Drap.) & 2 & 4 & - & - & - & - & - & 1 \\
\hline Vertigo pygmaea (Drap.) & - & - & - & - & 8 & 42 & - & - \\
\hline Truncatellina cylindrica (FÉR.) & - & - & - & - & 1 & - & - & - \\
\hline Pupilla muscorum (L.) & 2 & 1 & 18 & 1 & 6 & 71 & 2 & 1 \\
\hline Pupilla sterri (VorTH) & - & - & 3 & 2 & - & - & - & - \\
\hline Pupilla triplicata (STUD.) & - & - & - & - & 24 & 94 & 2 & - \\
\hline Vallonia costata (MüLL.) & - & 4 & 3 & 1 & 410 & 389 & 10 & - \\
\hline Vallonia enniensis GREDL. & - & - & - & - & - & - & 1 & - \\
\hline V.allonia pulchella (MüLL.) & 1 & - & 1 & 2 & 9 & 27 & 3 & - \\
\hline Chondrula tridens (MüLL.) & 32 & 28 & - & 6 & 104 & 30 & 30 & 2 \\
\hline Clausilia dubia Drap. & 1 ? & 4 & - & - & - & - & - & - \\
\hline Limacidae sp. (cf. Deroceras) & - & - & - & - & - & 17 & 6 & - \\
\hline ola fruticum (MüLL.) & 4 & 5 & - & 2 & - & 1 & 1 & - \\
\hline Helicella striata (MüLL.) & 27 & 17 & 12 & 2 & 193 & 127 & 49 & 1 \\
\hline Enomphalia strigella (DRAP.) & 3 & 4 & - & 1 ? & - & 1 & 1 & - \\
\hline Helicidae sp. div. & - & 1 & - & - & - & - & - & \\
\hline
\end{tabular}

In den Thanatozönosen MF 4 und MF 3 überwiegen gut erhaltene, größtenteils ganze Gehäuse, was beweist, daß diese Fauna nach ihrer Fossilisation sekundär nicht umgelagert wurde. Die anderen Funde sind durchaus fragmentarisch und machten offensichtlich einen längeren oder kürzeren Transpert durch. MF 1 aus dem oberen Lößkomplex besteht aus Konchylienfragmenten, die wahrscheinlich vorwiegend aus älteren fossilführenden Schichten sekundär umgelagert wurden.

Abgesehen vom vereinzelten Vrorkommen einiger Arten, stellen alle festgestellten Thanatozönosen einen verhältnismäßig geschlossenen Komplex von Gesellschaften dar, was eine ökologische Analyse der gesamten Fauna ermöglicht. Die Unterschiede zwischen den einzelnen Faunen werden wir erst zum Schluß der Gesamtanalyse hervorheben.

Für die festgestellten Thanatozönosen sind die Steppenarten Chondrula tridens (MülL.), Helicella striata (Müll.), Vallonia costata (MülL.) und Pupilla muscorum (L.) charakteristisch, zu denen sich weitere Elemente ähnlicher Ansprüche, z. B. Abida frumentum (DRAP.) in den unteren Schichten oder Truncatellina cylindrica (FÉR.) und Vertigo pygmaea (Drap.) in den obereri, gesellen. Ein wenig verschiedene Anspriiche haben die Schnecken Fruticicola fruticum (MüLI.) und Euomphalia strigella (Drap.), welche lichte Waldbestände bevorzugen, aber auch sonnige offene Standorte bewohnen können, namentlich trockene Strauchbestände. Besonders die Art E. strigella (Drap:) kann als kennzeichnendes Element der Waldsteppe betrachtet werden. Die übrigen Arten sind ökologisch weniger ausgeprägt und passen gut in den beschriebenen Rahmen mit Ausnahme des Einzelfundes von Vallonia enniensis GRD. in MF 2, die rezent zwar offene, aber immer recht feuchte Standorte bewohnt und auch fossil fast ausschließlich in Sumpffaunen vorkommt. Was die Vertreter der Gattung Succinea und Cochlicopa anbelangt, kann man nichts näheres sagen in Hinsicht auf die Unmöglichkeit einer genaueren Bestimmung. 
Das Hauptmerkmal aller Thanatozönosen ist das Fehlen charakteristischer feuchtigkeitsliebender und Waldarten. Die fossilen Schneckengesellschaften sind überwiegend von Steppencharakter, was sichere Beweise gibt, daß in ihrer Lebenszeit in der Umgebung von Leitmeritz eine Grassteppe vorhanden war. Die Funde von Fruticicola fruticum (MüLL.) und Euomphalia strigella (Drap.) zeigen, daß in dieser Steppe die Gehölze nicht völlig fehlten, sondern daß hier und da vereinzelte Gruppen von Sträuchern oder sogar Bäumen vorhanden waren. Der höhere Anteil beider Arten zeugt immer für ein häufigeres Vorkommen der Gehölze, was vor allem für die Thanatozönosen MF 8 und MF 7 gilt.

Während der Steppencharakter der Molluskenfauna auf den ersten Blick ersichtlich ist und einer eingehenderen Analyse nicht bedarf, ist die Antwort auf die Frage, wie warm das damalige Klima war, viel schwieriger. Die Melırzahl der festgestellten Arten ist nicht sehr temperaturanspruchsvoll und kann als eurytherm bezeichnet werden. Es handelt sich besonders um die Arten Pupilla muscorum (L.), Vertigo pygmaea (Drap.), Vallonia costata (MülL.) und $V$. pulchella (MüLL.), Clausilia dubia Drap., sowie die beiden großen Heliciden Fruticicola fruticum (MüLL) und Euomphalia strigella (DRAP.). Die letzte Art wird in Mitteleuropa oft für wärmeliebend gehalten; diese Ansicht ist jedoch nicht richtig, wie man sich leicht aus dem Verlaufe ihrer Nordgrenze in Osteuropa überzeugen kann (vgl. Licharev \& Rammelmayer 1952). Ahnlich ist der Fall auch bei den typisch xerothermen Arten Chondrula tridens (MüLL.) und Truncatellina cylindrica (FÉR.), die stets warmtrockene Standorte bewohnen. Wenn wir aber ihre Verbreitung in Nordosteuropa in Betracht ziehen, sehen wir, daß Ch.tridens (MüLL.) noch das Gebiet am Ural-Fluß erreicht, also einen Raum, wo die Frostperiode bis 6 Monate dauert und die durchschnittliche Januartemperatur zwischen $-10^{\circ} \mathrm{C}$ und $-15^{\circ} \mathrm{C}$ schwankt. Beide Arten ertragen also ein recht rauhes winterkaltes Klima. Auf den ersten Blick paß3t in den Rahmen der beschriebenen Schneckengesellschaft nicht Pupilla triplicata (STUD.), eine meridionale Art, die heute xerotherme Felsen in wärmsten Gebieten Mittel- und Südeuropas bewohnt. Wenn man aber in Betracht zieht, daßs sie in den Westalpen am Kalk bis in die alpine Stufe steigt und daß sie in der letzten Zeit vielmals im Löß in Gesellschaft von so kälteliebenden Arten wie Columella columella (BENZ) gesammelt wurde, muß man mit der Möglichkeit rechnen, daß sie unter bestimmten Bedingungen auch rauhes Klima gut ertragen kann. Helicella striata (MüLL.), eine häufig vorkommende Lößart, die heute in den Steppengebieten Mitteleuropas lebt, kann als Beispiel dienen, wie kritjsch man die klimatische Auswertung einzelner Mollusken auf Grund ihrer rezenten Ökologie durchführen muß. Heutzutage erscheint $H$. striata (MüLL.) als typisch wärmeliebende Art, während sie im Pleistozän ein geläufiges Mitglied von Gesellschaften war, deren Gepräge zweifellos kühl ist. Ein eingehendes Studium der Lebensansprüche dieser Art in der rezenten Zeit zeigt aber klar, daß sie an wenig entwickelte, stark kalkhaltige Böden von Syrosem- oder Protorendsina-Charakter gebunden ist und allmählich ausstirbt. Es handelt sich um eine ausgeprägt trockenliebende Art, die heute geeignete Lebensbedingungen nur in xerothermen Gebieten finden kann, ist aber nicht durch die höhere Temperatur dieser Gebiete beeinflußt, sondern durch die günstigen Feuchtigkeits-- und Bodenverhältnisse. Wenn wir diese Ansprüche mit den Umweltsverhältnissen der Lößphasen vergleichen, sehen wir, daß die Bedingungen in diesen Zeitabschnitten für H. striatı (MüLL.) optimal sein müßten.

Es bleibt übrig, noch einige Worte über Abida frumentum (DrAp.) zu sagen, welche die einzige wärmeliebende Art ist, die im Leitmeritzer Profil festgestellt wurde. Rezent ist Abida frumentum (DrAP.) ein rein xerothermes Element, dessen Nordgrenze am Südrand der Norddeutschen und Polnischen Tiefebene verläuft; Osteuropa wird nicht erreicht. Sie ist auch mikroklimatisch viel anspruchsvoller als z. B. Pupilla triplicata (STUD.), was sich klar aus ihrer beschränkten Verbreitung im Gebirge ergibt. Im Pleistozän ist sie vor allem in den Warmzeiten verbreitet, namentlich in den altpleistozänen (häufiges Mitglied der biharischen Faunen). Im Waagtal wurde sie aber verstreut auch im Löß fest- 
gestellt, hauptsächlich im Altwürm (W 1-Löß in Moravany, vgl. AMBrož-LožEK-ProšEK 1952). Dieser Fund belegt, daß unter außerordentlich günstigen Umständen auch diese Art in den Lößfaunen vorkommen kann, allerdings nur in den wärmsten, die nur in bestimmten beschränkten Abschnitten der Lößkomplexe zu finden sind, besonders im Altwürm, in welches auch die betreffenden Schichten des Leitmeritzer Profils eingereiht werden.

Aus der Analyse ergibt sich, daß die Schneckenbestände des Leitmeritzer Profils, namentlich die Faunen der Humushorizonte, a us Steppenarten mit geringer Beimischung von Waldsteppenschnecken bestehen, die auch rauhes Klima mit langen kalten Wintern ertragen können.

Wie bereits erwähnt, haben alle Thanatozönosen ein ziemlich einheitliches Gepräge und unterscheiden sich voneinander nur in Details.

Die Fauna der Deckschichten des unteren Bodenkomplexes (MF 8 und 7) zeichnet sich durch auffallend niedrigen Anteil der Arten aus den Gattungen Pupilla und Vallonia aus; Vertigo pygmaea (Drap.) und Pupilla triplicata (STUd.) fehlen hier völlig. Dagegen wurden aber die Arten Abida frumentum (Dr.s.), Clausilia dubia Drap. und ein ziemlich hoher Anteil von Fruticicola fruticum (MüLL.) und Euomphalia strigella (DRAP.) festgestellt, was für ein milderes Klima und einen Waldsteppencharakter der Landschaft zeugen darf.

In den hangenden nichthumosen Schichten (MF 6 und 5) macht sich eine beträchtliche Verarmung der Fauna bemerkbar; auch der Anteil von Chondrula tridens (MüLL.) sinkt auffällig, aber der Pupillen-Anteil steigt. Hier erscheint auch Pupilla sterri (VTr.), die in anderen Schichten nicht gefunden wurde. Die Thanatozönosen MF 6 und 5 sind den Lößgesellschaften am nächsten verwandt. Man kann sie als Zeugen der Austrocknung und Abkühlung, sowie des Steppencharakters der Umgebung betrachten.

Wie bereits erwähnt, zeichnen sich die oberen Humushorizonte (MF 4, 3 und 2) durch starkes Vorkommen der Arten aus den Gattungen Vallonia und Pupilla, besonders P. triplicata (Stud.), sowie von Vertigo pygmaea (Draf.) aus. Die Fauna des autochthonen unteren Humusbodens (MF 4) ist von reinem Steppencharakter und beweist, daß in der Umgebung eine Grassteppe ohne Gehölze vorhanden war. Interessant ist der Fund von Truncatellina cylindrica (FËr.), die man zu den typischen Elementen der pleistozänen Warmzeiten zählen muß und die der Lößfauna völlig fremd ist.

Die Fauna des nächst höheren Humusbodens (MF 3) ist durch den auffällig erhöhten Anteil von Pupillen und Vertigo pygmaea (Drap.) gekennzeichnet. Die Einzelfunde von Fruticicola fruticum (Müll.), Euomphalia strigella (DRAP.), Succinea sp., sowie das verhältnismäßig häufige Vorkommen der Nacktschnecken (Deroceras?) zeigen, daß sichtlich eine geringe Steigerung der Feuchtigkeit stattgefunden hat und daß in die Steppe Gehölze, wahrscheinlich Gruppen von Sträuchern, vorgedrungen sind. Ein ähnliches Gepräge hat auch MF 2, in der aber der Anteil von Pupillen und Vallonien stark gesunken ist. Von besonderem Interesse ist der Einzelfund der feuchtigkeitsliebenden Vallonia enniensis GRD., welche möglicherweise in die Schicht sekundär transportiert worden ist. Für diesen Transport zeugt nicht nur die fragmentarische Erhaltung der Konchylien in MF 2, sondern auch das häufige Vorkommen von Turon-Fossilien in der Schicht C 5.

Die spärlichen Funde aus dem oberen Lößkomplex (MF 1) werden hier in Hinsicht auf ihre allochthone Herkunft nicht näher analysiert.

Die Antwort auf die Frage, wie das Klima in der Lebenszeit der beschriebenen Molluskenfaunen, besonders während der Bildung der beiden oberen Humusböden (D 1 und D 3), beschaffen war, ergibt sich aus dem Vergleich der Ergebnisse unserer Analyse mit dem Charakter der Lößgesellschaften und der hochinterglazialen Faunen. Fossile Molluskenfaunen, die den Thanatozönosen MF 4, 3 und 2 aus Leitmeritz entsprechen, wurden in tschernosemartigen Böden, die wahrscheinlich dem Göttweiger Interstadial entsprechen, 
in Zájezd bei Buštěhrad (Ložex 1954), Sedlec bei Kuttenberg (ZÁRuba \& Hromada 1950) und Podbaba (LožEx 1955) gefunden. Aus dem wohlbekannten Profil in der Ziegelei von Unter-Wisternitz stammt ein Einzelfund von Chondrula tridens (MüLL.), die von F. ProšEK in dem mittleren Humushorizont entdeckt wurde. Auch im Waagtal führen die Schichten des Göttweiger Interstadials Molluskenfaunen, und zwar in Moravany (AмBrožLožEk-Prošex 1952, und Ivanovce (LožEK 1955). Spärliche Funde sind auch aus Zamarovce (ProšEk \& Ložek 1955) und Sered' (BÁRta 1957) bekannt. Alle erwähnten Funde sind in einer Übersichtstabelle (S. 90) zusammengefaßt, aus welcher sich ihr einheitlicher Charakter ergibt. Nur die Faunen aus dem Waagtal sind artenreicher und haben auch cinige Waldbewohner, was mit dem wärmeren und feuchteren Klima dieser Gegend zusammenhängt, wo auch die fossilen Böden in einer abweichenden Fazies entwickelt sind.

II. Tabelle der Molluskenfunde aus dem Göttweiger Interstadial

\begin{tabular}{|c|c|c|c|c|c|c|c|c|c|c|c|}
\hline \multirow{2}{*}{$\begin{array}{l}\text { Ókologische } \\
\text { Charak- } \\
\text { teristik }\end{array}$} & \multirow{2}{*}{ Molluskenarten } & \multicolumn{10}{|c|}{ Fundstellen } \\
\hline & & 1 & 2 & 3 & 4 & 5 & 6 & 7 & 8 & 9 & 10 \\
\hline$S !$ & Abida frumentum (DRAP.) & - & - & - & - & - & + & + & - & - & t \\
\hline $\mathrm{O}$ & Vertigo pygmaea (DrAP.) & + & - & - & - & - & + & - & - & - & - \\
\hline $\mathrm{S}$ & Truncatellina cylindrica (FÉR.) & + & - & - & - & - & - & - & - & - & - \\
\hline $\mathrm{O} ! !$ & Pupilla muscorum (L.) & + & + & - & - & - & + & + & - & - & \\
\hline$S ! !$ & Pupilla triplicata (STUDER) & + & + & - & - & - & + & + & - & - & - \\
\hline \multirow[t]{2}{*}{$S ! !$} & Pupilla sterri (VoIтн) & - & - & - & - & - & - & + & - & - & \\
\hline & Orcula dolium (Drap.) & - & - & - & - & - & + & - & - & + & - \\
\hline \multirow[t]{2}{*}{$\mathrm{O} ! !$} & Vallonia costata (Müller) & + & + & - & - & - & + & - & - & - & \\
\hline & Vallonia enniensis GREDLER & + & - & - & - & - & - & - & - & - & \\
\hline $\mathrm{O}$ & Vallonia pulchella (MüLL.) & + & + & - & - & - & + & - & - & - & \\
\hline$S !$ & Chondrula tridens (MüLLER) & + & + & + & + & + & + & + & + & + & 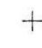 \\
\hline \multirow{2}{*}{ W } & Cocblodina laminata (MTG.) & - & - & 一 & - & - & + & $+?$ & - & - & - \\
\hline & Clausilia dubia Drap. & - & - & - & - & - & + & - & - & - & - \\
\hline \multirow[t]{2}{*}{ W } & Clausilia pumila C. PFr. & - & - & - & - & - & + & - & - & - & \\
\hline & Limacidae sp. & + & + & - & - & - & + & + & - & - & \\
\hline WS & Fruticicola fruticum (MÜLLER) & + & + & - & - & - & - & + & + & - & 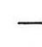 \\
\hline$S: !$ & Helicella striata (MüLLER) & + & + & + & + & - & + & + & - & - & T \\
\hline W & Monachoides vicina (Rossm.) & - & - & - & - & - & - & + & - & - & \\
\hline WS & Euomphalia strigella (Drap.) & + & - & - & - & - & + & + & - & - & \\
\hline ! & Arianta arbustorum (L.) & - & - & - & - & - & - & - & + & + & \\
\hline
\end{tabular}

Erläuterungen:

Ookologische Charakteristik: $\mathrm{S}=$ Steppe, warmtrockene waldlose Standorte; O = offene Biotope verschiedener Art (feuchte Wiesen bis sonnige Felsen und Steppen); WS = Waldsteppe, lichter Wald; W = Wald; ! ! = Lößarten; ! = lokale Mitglieder der Lößfauna.

Fundstellen: 1 Leitmeritz (MF 4, 3,2). - 2 Zájezd bei Buštěhrad, fossiler Tschernosem und oberste Schicht des liegenden Lösses (LožEK 1954b). - 3 Podbaba bei Prag, fossiler Tschernosem (LožEK 1955). - 4 Sedlec bei Kuttenberg, fossile Rendsina (Záruba \& Hromada 1950). 5 Unter-Wisternitz, Ziegelei, mittlerer Humushorizont (leg. Prošex). - 6 Moravany im Waagtal, fossiler schwarzerdeähnlicher Boden (Aмвrož-LožEK-ProšEK 1952). - 7 Ivanovce im Waagtal, Skala, oberste Schicht des Lösses im Liegenden des fossilen Bodens mit SzeletaKultur (LožEK 1955). - 8 Ivanovce, Járok, Oberfläche eines fossilen Tschernosems (LožEK 1955). - 9 Zamarovce im Waagtal, Oberfläcte der fossilen Braunerde mit Szeletien (ProšEK \& Ložex 1955). - 10 Sered im Waagtal, Sanddünen bei Mača, fossile Pararendsina auf kalkhaltigem Dünensand (BáRTA 1957). 
Aus der Tabelle ist ersichtlich, $\mathrm{da} ß$ es sich durchaus um klimatisch wenig anspruchsvolle Faunen handelt, die größtenteils aus Steppenarten bestehen und die lokal an manche rezente, bzw. postglaziale Molluskenzönosen stark erinnern können, von denen aber sie sich stets durch das Fehlen einiger echter wärmebedürftiger Arten wie Zebrina detrita (MülL.), Vitrea inopinata (UL.), Helicella obvia (HTM.), Cepaea vindobonensis (FÉr. oder Cecilioides acicula (MüLL.) unterscheiden. Viel auffallender ist der Unterschied im Vergleich mit den Schneckengesellschaften des Letzten Interglazials, die aus den Lößprofilen von Sedlec bei Prag, Letky, Jenerálka, Předmostí und Zamarovce bekannt sind. Die interglazialen Thanatozönosen sind viel artenreicher und zeichnen sich durch die vollkommene Vorherrschaft der feuchtigkeitsliebenden und temperaturanspruchsvollen Waldarten aus, von denen einige heute nur im südlichen Europa leben (Helicigona banatica (Rssm.), Soosia diodonta FÉr.), Aegopinella ressmanni (West.) usw. (vgl. Ložek 1955). Die Steppenkomponente ist nur sehr schwach vertreten und zwar auch in Gebieten, deren Fauna im Postglazial und in der heutigen Zeit vorwiegend von Steppencharakter ist. Das Letzte Interglazial ist also beträchtlich wärmer und hauptsächlich feuchter als die Gegenwart, während das Göttweiger Interstadial viel trockener, kontinentaler und im Durchschnitt auch deutlich kälter ist. Dieser Unterschied ergibt sich bereits aus einem flüchtigen Anblick auf die Faunenlisten und deren Artenreichtum. Während die Artenzahl in den interstadialen Malakozönosen gegen 10 beträgt, bestehen die letztinterglazialen Faunen üblicherweise aus 30-50 Arten. Die Gebiete, wo im Postglazial die Waldsteppe vorherrschte, waren im Letzten Interglazial von zusammenhängenden Urwäldern bedeckt, wogegen im Göttweiger Interstadial die Steppe die Hauptrolle im Landschaftsbild spielte.

Die Gesellschaften des Göttweiger Interstadials sind scheinbar den Lößthanatozönosen recht ähnlich. Arten wie Helicella striata (MüLL.), Vertreter der Gattung Pupilla und Vallonia costata (MüLL.) kommen geläufig im Löß vor. Wenn man aber eine eingehende Analyse durchführt, treten sofort einige grundsätzliche Unterschiede hervor. In der Fauna des Interstadials fehlt eine ganze Reihe typischer Lößarten, wie Vertigo parcedentata (A. BR.), Columella columella (Benz), Putilla loessica LžK, Vallonia tenuilabris (A. BR.), Lößformen aus den Kreisen von Pupilla muscorum (L.) und Trichia hispida (L.) usw. Im Gegenteil finden wir in den interstadialen Faunen einige Schnecken, die der Lößfauna völlig fremd sind ${ }^{2}$ ), wie Truncatellina cylindrica (FÉr.), Vallonia pulchella (MüLL.) und Vertigo pygmaea (Drap.). Formen der Arten, die sowohl in Löß, als auch in Nichtlößablagerungen vorkommen, sind im Göttweiger Interstadial immer mit den warmzeitlichen Formen und nicht mit den Lößformen der betreffenden Arten identisch. Dieser Unterschied ist nicht zu unterschätzen, da er in manchen Fällen recht auffallend ist, z. B. bei Pupilla muscorum (L.).

Wenn man die Ergebnisse der Analyse der Leitmeritzer Molluskenfauna zusammenfaßt, sieht man, daß die Thanatozönosen MF 4, 3 und 2 einem Zeitabschnitt von rauhem Festlandsklima mit warmen Sommern und langen kalten Wintern entsprechen, das sich sowohl vom warmfeuchten Klima des Interglazials, als auch vom trockenkalten Klima der Stadiale scharf unterscheidet. Diese Bedingungen sind typisch für ein Interstadial. Die Fauna aus den unteren Schichten (MF 8 und 7) zeugt für cin zwar mäßig kaltes, aber feuchteres Klima, welches wahrscheinlich am Ende des Interglazials vorgeherrscht hat, während MF 6 und 5 bereits den Übergang zu kalten Lößgesellschaften darstellt.

2) Unter dem Begriff „Lößart“ muß man nur solche Arten verstehen, die im primären Löß gesammelt wurden. In der Literatur, namentlich in der älteren, werden unter dem Termin „Lößarten" oft einfach alle Funde aus den Lößkomplexen angeführt, also auch die Funde aus fossilen Podenkomplexen, Fließerde- und Hangablagerungen, die mit der eigentlichen Lößfauna nichts zu tun $\mathrm{zu}$ haben brauchen. 


\section{Stratigraphische Würdigung des Profils}

Auf Grund der in der Übersichtstafel (I) angeführten Daten und der Molluskenfunde wurde die stratigraphische Wertung des Profils durchgeführt. Wir werden hier kurz erklären, welche Merkmale zur sedimentologischen und pedologischen Würdigung der einzelnen Schichten benïtzt wurden, wobei wir die Aufmerksamkeit vor allem auf stratigraphisch wichtige oder schwer zu würdigende Schichten richten werden. Eine eingehende klimatologische Deutung der festgestellten Daten kann im Rahmen dieser vorwiegend stratigraphischen Studie nicht durchgeführt werden, wird aber in der tschechoslowakischen Fachliteratur später erscheinen.

Die älteste stratigraphische Einheit im untersuchten Profil ist der Basislöß (G). Der primäre Charakter dieser Lößablagerung ist durch alle festgestellten Gefüge-Merkmale, sowie durch das relativ niedrige Volumengewicht und den hohen Glimmergehalt mit Sicherheit erwiesen. Dieser Löß zeichnet sich durch einen hohen Karbonatgehalt aus, der an die Mergel- und Plänerpartikel in der Feinstaubfraktion gebunden ist, die z. B. im Bereiche der Korngrößen 0,01-0,1 mm in den Proben L 22 und 94 gegen 30\% des Materials bilden. Der Löß ist gleichmäßig gefärbt und zwar ein wenig rostiger als die Hauptwürmlösse im oberen Abschnitt des Profils. Er enthält geringmächtige Einlagerungen von kantigem Plänerschutt, dessen Oberfläche von einer entkalkten Verwitterungsrinde bedeckt ist. Auch der niedrige $\mathrm{CaCO}_{3}$-Gehalt in der Fraktion $0,1-0,2 \mathrm{~mm}$ in der Probe L 22 zeugt für die Korrosion des Karbonats in den oberen Schichten dieser Lößablagerung. Die Korrosion verursachte den Zerfall feiner Mergelpartikel und eine Steigerung des Tongehaltes in der betreffenden Schicht (vgl. den niedrigen Wert von II/I · 100, Spalte 25). Diese Erscheinung, die gewissermaßen dem Verlehmungsprozeß verwandt ist, konnte selbstverständlich aus dem Löß bei weitem nicht den gesamten Karbonatgehalt entfernen. Die Plänerschutteinlagerungen können als Zeugen schwacher Fließbewegungen während der Lößbildung gedeutet werden. Das Material ist verhältnismäßig feinkörnig, und man kann voraussetzen, daß die Tragkraft des Windes etwa die gleiche war wie im Falle der Lösse B 3-6. Die pedogenetischen Beziehungen zum humosen Boden im Hangenden des Lösses sind recht kompliziert und werden bei der Beschreibung dieses Bodens behandelt.

Der untere Komplex der humosen Böden (F) liegt auf dem Basislöß (G), von dem er durch eine scharfe Grenze getrennt ist. Der untere sattgefärbte humose Lehm, der krümelig und in den oberen Partien staubig zerfällt, ist durch Fließerscheinungen weitgehend deformiert. In die Mitte des Humushorizontes wurde eine Schliere von grauweißem, stark kalkigem Lehm verschleppt, die dem ursprünglichen Ca-Horizont dieses Bodens entspricht. Der Boden ist weiter durch eine senkrechte, ca. 0,5 m tiefe Spalte gestört, die bis in den liegenden Löß vordringt und von feinstaubig zerfallendem humosem Material ausgefüllt ist. Der Dünnschliff aus der Probe 90 zeigte ein typisches Schwammgefüge des Bodens, die gleichmäßig verteilte Humussubstanz in der Mullform und unbewegte Brauneisen- und Tonsubstanz. In Hinsicht auf den hohen Kalkgehalt und die relativ geringe Mächtigkeit kann dieser Boden als Mullrendsina bezeichnet werden. Die regelmäßige Struktur und die sortierte Körnung bezeugen, daß der Boden auf Lößmaterial entstanden ist. Das Auftreten des neugebildeten Karbonats und die Korrosion des Plänerschutts im Schichtenkomplex G 2 können nicht durch den pedogenetischen Prozeß erklärt werden, bei dem sich die oben besprochene Mullrendsina (F 3) entwickelt hat. Der durch das Erdfließen verschleppte deutlich entwickelte $\mathrm{Ca}$-Horizont dieser Rendsina und die Karbonatausscheidungen in den Wurzelröhrchen (Probe 91) zeigen, daß die mit der Entwicklung des Bodens F 3 verknüpfte Karbonatbewegung nicht tief reichte. Demgegenüber sind aber die gröberen Bruchstücke des Kalkmergels im Abschnitt G 1-2 deutlich korrodiert, und das Karbonat ist in die obere Lage von G 3 hinabgetragen, wo es in Schlieren und in den Wurzelröhrchen ausgeschieden ist. Die rostige Tönung der oberen Lößlage steht mit dem beschriebenen Prozeß in Zusammenhang. Man muß also in den Schichten F 3 - G 3 zwei 
zeitlich verschiedene bodenbildende Prozesse voraussetzen, von denen der erste zur schwachen Entkalkung der Lößoberfläche $G$ führte (Beginn der Braunerdebildung?), ohne mit der Entstehung des höheren Humushorizontes verbunden zu sein. Der zweite ließ eine Mullrendsina entstehen, deren Ca-Horizont die früher entkalkte Zone G 1 teilweise eingenommen hat. Die Mullrendsina entwickelte sich wahrscheinlich auf einer neu sedimentierten Schicht von Lößmaterial, das hierher von benachbarten Aufschlüssen im unverwitterten Löß umgelagert wurde. Die Gefügemerkmale, welche die Art dieser Sedimentation feststellen ließen, wurden durch die zweite Bodenbildung verwischt. Aber in Hinsicht auf die geringe Mächtigkeit der kritischen Lage und die Tatsache, daß die Quarzkörner größtenteils eine glänzende Oberfläche besitzen und hier und da Spuren einer verwischten äolischen Bearbeitung zeigen, sind wir der Meinung, daß die Schicht durch Abspülung sedimentiert wurde. Zur Illustration des Unterschieds in der Zusammensetzung der Fraktion 0,1-0,2 mm bei den Proben L 22 (Schicht G 1) und 90 (Schicht F 3) soll folgende Tabelle (III) dienen:

\begin{tabular}{c|c|c|c|c|c|c|c|c}
\hline Probe & Quarz & Feldspat & $\begin{array}{l}\text { Kalk- } \\
\text { detritus }\end{array}$ & $\begin{array}{l}\text { Toramini- } \\
\text { feren und } \\
\text { Spongien- } \\
\text { nadel }\end{array}$ & Biotit & Muskovit & Limonit & $\begin{array}{l}\text { Akzes- } \\
\text { sorien }\end{array}$ \\
\hline 90 & $56 \%$ & $8 \%$ & $20 \%$ & $1 \%$ & $5 \%$ & $0,5 \%$ & $6 \%$ & $6 \%$ \\
22 & $61 \%$ & $16 \%$ & $4 \%$ & $4 \%$ & $0,5 \%$ & $2 \%$ & $4 \%$ & $\begin{array}{c}4 \% \\
\text { (einschl. } \\
\text { Bein- } \\
\text { detritus) }\end{array}$ \\
\hline
\end{tabular}

Die Schichten F 1-2 sind sandig-aleuritische Mergel unhomogener Körnungsart, deren untere Partien durch Fließerscheinungen deformiert wurden. Im oberen Abschnitt dieses Komplexes ist eine Protorendsina (F 1) entwickelt, die in kleine Aggregate zerfällt. In Hinsicht auf den hohen Tonanteil ist auch der Karbonatgehalt beträchtlich hoch. In diesem Komplex tritt ziemlich häufig kleiner kantiger, unverwitterter Plänerschutt auf. Beim Studium der Dünnschliffe und bei den Binokular-Untersuchungen wurden mit Ausnahme des erhöhten Glimmeranteils keine Anzeichen der äolischen Herkunft dieser Erden festgestellt. Insbesondere wurden keine Partikel von tonig verwittertem Mergel beobachtet, die für die „wcißen“ Lösse kennzeichnend sind (vgl. z. B. die Horizonte E 1 und C 2). Deshalb ist es wahrscheinlich, daß die Schichten durch Abspülung oder Solifluktion sediment,ert wurden. Ein dichtes Netz feiner Wurzelröhrchen beweist, daß der Boden F 1 von einem dichten Rasen bedeckt war. Der Horizont F 2 enthält die älteste im Profil festgestellte Molluskenfauna (MF 8), die einer mäßig trockenen Steppenlandschaft mit verstreuten Gehölzen angehört und einem kälteren Klima entspricht.

Im Hangenden des Bodenkomplexes F liegt eine Schichtenfolge von abgeschwemmten Ablagerungen, die von Löß überlagert werden (E). Die mergelige basale Schicht dieses Komplexes hat noch einen ziemlich hohen Humusgehalt und besteht größtenteils aus umgelagertem Material des liegenden Bodens. Durch den Vergleich der Schichten E 5 und F 1 treten die Unterschiede zwischen dem autochthonen Boden und dem Bodensediment deutlich hervor. Man findet sie in den Struktur- und Textureigenschaften und in der Verteilung der Poren, die dem Volumengewicht entspricht. Die autochthonen Böden haben im A-Horizont immer ein niedrigeres Volumengewicht als die Bodensedimente (=umgelagerte Böden). Der Dünnschliff aus der Schicht F 1 (Probe 87) zeigte ein regelmäßig verzweigtes System feiner Poren und eine deutliche Bildung von Feinaggregaten, deren Größe etwa 0,1 mm beträgt. An dieser Aggregatbildung beteiligen sich die Humusstoffe. Demgegenüber erscheint im Horizont E 5 (Probe 85) die Grundmasse als vollkommen verkittet mit vereinzelten, nicht zusammenhängenden Poren ohne jegliche Zeichen der Aggregatbildung. Man kann hier die autochthonen Ausscheidungen von rötlichbraunem Limonit 
deutlich verfolgen. Der Mergel E 4 wurde durch die Abspülung sedimentiert ebenso wie die liegende Schicht E 5. Die Grenzen der Horizonte sind recht scharf, die Polyederabsonderung ist durch Volumenänderungen bei Feuchtigkeitsschwankungen verursacht. Von Interesse ist die sandige Lage E 3 mit einer Plänerschutteinlagerung. In ihrem unteren Abschnitt besteht sie aus scharf begrenzten Bändern von Sand mit einer maximalen Korngröße von $1 \mathrm{~mm}$, die mit homogenen feinporigen Mergelschichten abwechseln. In Hinsicht auf das laufende Herabsinken des Wendepunktes und des Volumengewichtes in der Richtung nach oben, was einem schnell ansteigenden Porengehalt entspricht, können wir den Horizont E 3, wenigstens seinen oberen Abschnitt, als grobkörnige Lößbasis betrachten (vgl. auch das Gefüge). Die relativ feine mergelige Einlagerung E 2 wurde wahrscheinlich unter Mitwirkung von Wassertransport sedimentiert. Im Dünnschliff (Probe 83) aus dieser Schicht sieht man regelmäßig verteilten Quarz- und Kalkstaub und Feinsand in der mergeligen Grundmasse mit vereinzelten Poren, deren Größe etwa 0,03 mm beträgt. Der gröbere Detritus bildet selten undeutlich begrenzte wolkige Akkumulationen unregelmäßiger Form. Dieses Material schließt eine große Mienge Foraminiferen und Spongiennadeln ein, die aus den kretazischen Gesteinen des Felsgrundes freigelegt wurden.

Der lichtgelbe aleuritische Mergel E 1 weist bereits unstreitige Merkmale eines primär sedimentierten Lösses auf. Das sind: die regelmäßige verkittete Einzelkornstruktur, das niedrige Volumengewicht, die regelmäßig verteilten miteinander zusammenhängenden Poren und der deutlich erhöhte Glimmeranteil im Vergleich mit dem Liegenden. Die Schicht E 1 gehört zu den sogenannten weißen Lössen, die aus äolisch transportierten Partikeln der Kreidemergel bestehen und im Bereiche des Böhmischen Kreideplateaus ziemlich häufig zu finden sind. Näher werden diese Sedimente bei der Schicht $\mathrm{C} 2$ beschrieben. In Hinsicht auf eine gewisse Entkalkung und den Zerfall der primären Mergelkörner steigt in diesem Löß der Tonanteil sekundär von unten nach oben (zum Boden D 3). Gleichzeitig sinkt der $\mathrm{CaCO}_{3}-$ Gehalt im Feinschlamm ab. Wir können also schließlich den Komplex $\mathrm{E}$ als eine durch Abspülung sedimentierter Ablagerungen entstandene Schichtenfolge charakterisieren, welche oszillierend in einen grobkörnigen sandigen Löß übergeht. Wir bemerken, daß etwa $50 \mathrm{~m}$ in südöstlicher Richtung dieser Komplex mächtiger wird und sich in einen Komplex deutlich geschichteter, durch Wasser sedimentierter Sande mit Mergelzwischenschichten untergliedert, die im Hangenden gleichfalls in Löß übergehen. Die Bildung dieses Schichtenkomplexes setzte eine spärliche Pflanzendecke voraus, die eine umfangreichere Bodenabtragung nicht verhindern konnte. Das Klima war anfangs feucht, später wurden die feuchten Phasen (wahrscheinlich durch wolkenbruchartige Regen charakterisiert) durch längere Trockenperioden unterbrochen, während deren der Boden austrocknete und an der Oberfläche zerfiel, so daß der Wind den Bodenstaub leicht verwehen konnte. Im Komplex E wurden die Molluskenfaunen MF 7, 6 und 5 festgestellt, die zwar der älteren MF 8 recht ähnlich sind, doch aber für eine allmähliche Klima verschlechterung (d. h. Abkühlung und Austrocknung) zeugen.

Im Hangenden der beschriebenen Serie befindet sich ein Komplex von zwei ausgeprägten fossilen Böden (D). Der untere ist der mächtigste und am besten entwickelte Boden im ganzen Profil. Seine durchschnittliche Mächtigkeit beträgt $55 \mathrm{~cm}$. Er liegt auf dem Löß E 1, dessen oberste Schicht, wie bereits erwähnt, Spuren der bodenbildenden Prozesse aufweist. Die oberen $5-10 \mathrm{~cm}$ entsprechen einer gleichmäßig schwarzgrau gefärbten, staubig zerfallenden und teilweise entkalkten Zone, die allmählich in einen ein wenig lichteren bräunlichen Horizont mit Krümelstruktur übergeht, in dessen oberer Schicht zahlreiche Konchylien vorkommen (MF 4). Diese Zone hat eine schwache rostigbraune Tönung und deutliche unscharf begrenzte limonitische Inseln. Unter dieser Zone verläuft eine gelbbraune Übergangszone von gröberer krümeliger Struktur mit schwarzen regelmäßig verteilten Aggregaten, die nach unten alimählich verschwinden. Der staubige Horizont (D) füllt bis $3 / 4 \mathrm{~m}$ tiefe Spalten aus, die bis in das Liegende des Bodens reichen. 
Das Material dieser Spalten und ähnlicher Bildungen bei allen Böden im Leitmeritzer Profil unterscheidet sich von seiner Umgebung hauptsächlich durch sein Gefüge. Die Gefügeänderung entlang den Spalten konnte durch verschiedenste mechanische Vorgänge verursacht werden. Das mikroskopische Bild dieses Bodens stimmt mit einer Mullrendsina im Stadium der beginnenden Degradation überein. Es ist wahrscheinlich, daß während der Bodenbildung eine intensive Tätigkeit der Bodentiere stattgefunden hat; sichere Wurmkotstrukturen wurden aber nicht gefunden. Wurzelröhrchen von Kräutern und zahlreiche Schalen von Steppenmollusken beweisen, daß der Boden unter einer Grassteppe entstanden ist, was mit den Ergebnissen der petrographischen Analyse in voller Ubereinstimmung steht.

Es folgt der zweite große Boden des behandelten Komplexes (D 1), der in seiner Struktur der bereits beschriebenen Mullrendsina recht ähnelt, aber flachgründiger und weniger humos ist. Auch hier wurde eine Migration des Eisenhydroxyds nicht beobachtet. Die Schneckenfauna (MF 3) wurde in der staubigen Oberflächenzone gesammelt. Sie ist der MF 4 ziemlich ähnlich. Die lichte, stark versinterte Erde an der Basis dieser Mullrendsina mit Ca-Horizont ist wahrscheinlich abgespült, wofür ihre Unsortiertheit, das sichtlich primär prädisponierte feine Korn und das Fehlen der Poren zeugen. Man kann allerdings die Möglichkeit nicht ausschließen, daß es sich um eine Lage von weißem Löß handle, dessen Struktur durch die Bodenbildung verändert wurde. Dafür dürfte der erhöhte Glimmeranteil in der Korngrößenklasse unter 0,1 mm zeugen. Das Vorhandensein zahlreicher Konchylien zeugt für ein reges Bodenleben und für das Vordringen der Gehölze in die Steppe.

Das mikroskopische Bild beider Böden des D-Komplexes ist ähnlich: es zeigt eine deutliche Aggregatbildung und ein regelmäßiges Schwammgefüge. Der Humus in der Mullform ist in der tonigen Grundsubstanz ziemlich gleichmäßig verteilt, bildet aber lokale Schlieren und ist auch an der Oberfläche der Aggregate relativ konzentriert. Es gibt hier auch scharf begrenzte kleine Limonitkonkretionen sowie seltene Ausscheidungen von Manganolimonit in den Wurzelröhrchen. Im oberen Abschnitt des A-Horizontes hat die obere Rendsina (D 1 Dünnschliff aus der Probe 74) ein hohlraumarmes Gefüge mit seltenen Poren, eine nichtsortierte Körnung, seltene autochthone Schlieren von lichtbraunem Limonit und entlang den Spalten die beginnende Feinaggregatbildung. Ein ähnliches Gefüge, allerdings mit ausgeprägter Eisenausscheidung, kann an der Basis der Schicht D 3 vorausgesetzt werden.

Der folgende Komplex C ist durch die Sedimentation des weißen Lösses gekennzeichnet, die dreimal durch Entwicklung autochthoner Bodenhorizonte unterbrochen wurde. Der Komplex beginnt mit einer Fließerdelage, in der teilweise die oberen Partien der liegenden Rendsina (D 1) umgelagert sind. Höher enthält diese Lage kleinen Plänerschutt und geht in eine mergelige Erde mit deutlicher mikroskopischer Lößstruktur über. Im Dünnschliff wurden Partikel stark kalkigen Mergels zusammen mit Quarz- und Kalksteinkörnern, sowie mit kalkigen Kreidefossilien (Foraminiferen, Spongiennadeln) beobachtet; alle Körner in gleichmäßig sortierter Größe von 0,01-0,04 mm. Die Poren zwischen den einzelnen Körnern sind klein, regelmäßig, größtenteils aber nicht miteinander verbunden. Der Limonit ist in feinen, unscharf begrenzten Flocken ausgeschieden. Entlang den Spalten kann man die beginnende Feinaggregatbildung beobachten, die durch den Einfluß der hangenden Bodenbildung entsteht. Der Boden C 5, in dem die Molluskenfauna MF 2 festgestellt wurde, hat noch einen relativ hohen Humusgehalt $(25 \%)$ im Vergleich mit dem postglazialen Boden. Seine autochthone Position ist durch die Häufigkeit der Poren, die fortgeschrittene Aggregatbildung und das Vorhandensein eines CaHorizontes unstreitig bewiesen. Im Dünnschliff erscheint die Schicht C 5 als ein unregelmäßig feinporiger Boden mit feinsten Aggregaten und weißlichgrauen Schlieren dichten Gefüges, die durch das Karbonat imprägniert sind (wahrscheinlich sekundär vom Han- 
genden). Die Größe der Aggregate schwankt zwischen $0,1-0,8 \mathrm{~mm}$, der Mull ist regelmäßig verteilt. In einigen Spalten und Leitbahnen gibt es Ausscheidungen von Manganolimonit. Dieser Boden, welcher auch noch zu den Mulirendsinen gerechnet werden muß, geht nach unten über in eine feinkrümelige, ein wenig schwächer braungrau gefärbte Schicht, an deren Basis das Kalziumkarbonat in den Spalten und Wurzelröhrchen ausgeschieden ist. Sichere Zeichen der Tätigkeit der Bodenlebewesen wurden nicht festgestellt. Die Konchylien (MF 2) sind durchaus nur in Fragmenten erhalten, was für einen sekundären Transport zeugt. Sie zeigen aber, daß der Boden im trockenen Steppenklima gebildet wurde.

Der jüngere Boden C 3 ist unten krümelig, oben staubig. Seine Mächtigkeit ist relativ gering, Mollusken wurden nicht festgestellt. Die obere Grenze ist unscharf. Im Dünnschliff erscheint dieser Boden als sehr schwach entwickelt; die Aggregate sind undeutlich, dafür aber wurden schlierenartige Akkumulationen von Karbonat und Limonit beobachtet. Der Humus ist gleichmäßig verteilt. Der Boden kann als Protorendsina bezeichnet werden. Er ist durch kleine Spalten gestört, die sich durch ihr Gefüge und ihre Farbe von der Umgebung unterscheiden. Die Bildung dieser „Keile“ ist an mechanische Störungen entlang den Spalten gebunden, z. B. an abwechselndes Austrocknen und Feuchtwerden sie braucht nicht durch die Frosttätigkeit verursacht zu sein, und die Spalten sind mit den Frostkeilen nicht identisch.

Im Liegenden dieses Bodens ist die Schicht C 4, welche ein unhomogenes, hohlraumarmes Gefüge aufweist. Im Dünnschliff kann man primäre Schlieren der Tonsubstanz beobachten, die offenbar während der Sedimentation entstanden sind und Lagen von Quarzund Kalksteinschluff in der Korngröße 0,01-0,03 mm einschließen. Die mikroskopischen Poren sind selten; relativ häufiger sind die Wurzelröhrchen von $0,1-0,2 \mathrm{~mm}$ Durchmesser. Aus den erwähnten Tatsachen und in Hinsicht auf weitere Daten in der Tabelle ist ersichtlich, daß die Schicht C 4 wahrscheinlich durch Abspülung sedimentiert wurde.

Der Mergel C 2 ist ein ty pisches Beispiel des weißen Lösses. Er hat einen hohen Anteil von kalkigem Ton und nur wenig Staub; die ursprüngliche Korngrößenverteilung ist aber ganz anders gewesen. Im Dünnschliff ist das regelmäßig porige, aleuritische bis psammitische homogene Gefüge des Mergels deutlich sichtbar. Die Größe der Körner beträgt 0,01-0,08 mm, am häufigsten 0,03-0,05 mm; sie bestehen einerseits aus Quarz, Kalkstein, Feldspat, feinem Kalksandstein usw., andererseits aus Mergel. Die Mergelpartikel sind abgerundet und von gleicher durchschnittlicher Größe wie die Körner der anderen Minerale. Beim Schlämmen der Probe werden diese Körner allerdings zerstört, was das Ergebnis der Kornanalyse verzeichnẹt. Die Entstehung der böhmischen weißen Lösse ist an solche Gebiete gebunden, wo als Ausgangsgestein der Lößbildung feinkörnige Turonmergel in beträchtlichem Maße auftreten. Durch mechanische Verwitterung. vom Felsgrund abgesonderte Mergelsplitter wurden vom Winde auf kurze Entfernungen transportiert und zusammen mit festerem Mineraldetritus aus größerer Entfernung sedimentiert.

Zur Illustration der mineralogischen Zusammensetzung des weißen Lösses führen wir die perzentuelle Zusammensetzung der Fraktion 0,1-0,2 $\mathrm{mm}$ an, die bei der Kornanalyse der Probe L 9 isoliert wurde:

$\begin{array}{lr}\text { Quarz (größtenteils äolisch bearbeitet) } & 42 \% \\ \text { Feldspat (größtenteils nur schwach angewittert) } & 4 \% \\ \text { Kalkstein (Sandiger Kalkstein, Kalzit) } & 22 \% \\ \text { Foraminiferen und Spongiennadeln } & 13 \% \\ \text { Biotit (zum größten Teil baueritisiert) } & 8 \% \\ \text { Muskovit } & 4 \% \\ \text { Limonit } & 4 \% \\ \text { Akzessorien } & 3 \%\end{array}$


Von Interesse ist der niedrige Anteil der Feldspäte im Vergleich zum Quarz, dessen Summe in Hinsicht auf die Zusammensetzung der basalen Schichten auch beträchtlich herabgesunken ist. Darin spiegelt sich die lange und wiederholte Verwitterung der Aufschlïsse in den Terrassenschottern ab, von denen die Mehrzahl der Quarz- und Feldspatkörner stammt.

Der jüngste Boden des C-Komplexes (C 1) ist nur durch eine wenig dunklere Tönung gekennzeichnet. Er hat einen geringen Humusgehalt, ist aber schwach entkalkt, und das freigewordene Karbonat ist in seinem Liegenden wieder sekundär ausgeschieden. Die Aggregatbildung wurde nicht beobachtet. Dieser pedogenetische Prozeß kann als Initialstadium der "Braunerdebildung“ betrachtet werden.

Der Lößkomplex B zerfällt in zwei Hauptabschnitte. Der untere beginnt mit einem grobkörnigen weißen Löß (B 7), setzt in der mächtigen Solifluktionszone (B 6) mit häufigen Quarzgeröllen und Plänerschutt fort und endet im Lösse B 5 und B 3, dem eine nicht zusammenhängende Fließlage mit verstreuten Geröllen und Plänerschutt eingelagert ist (B 4 mit MF 1). In der Kornzusammensetzung erscheint der gesamte beschriebene Abschnitt als ein Löß. Es bleibt allerdings zu entscheiden, ob die Schichten B 5 und B 6 tatsächlich mit dem basalen weißen Löß B 7 zeitlich verbunden sind, da er eine stark abweichende Zusammensetzung aufweist. Unsere Würdigung wird von der Beobachtung unterstützt, daß die einzelnen Glieder dieser Schichtenfolge durch allmähliche Übergänge verbunden sind. Es ist sehr wahrscheinlich, daß das mächtige Erdfließen während der beginnenden äolischen Sedimentation die Verteilung der Ausgangs-Verwitterungsprodukte in der Umgebung des Profils, aus denen das Lößmaterial verweht wurde, völlig geändert hat. Die Mergel-Eluvien traten zugunsten der Schottersande der Elbeterrassen stark zurück. Demzufolge ist der Kalkgehalt der abgelagerten Lösse beträchtlich abgesunken; er erreicht nur noch die üblichen Werte zwischen $10-15 \% \mathrm{CaCO}_{3}$.

Die Möglichkeit; daß die Fließerdeschicht B 6 den liegenden Löß B 7, auf dem möglicherweise eine unbekannte Bodenbildung vorhanden war, diskordant abschneidet, kann auf Grund der zur Verfügung stehenden Daten nicht sicher ausgeschlossen werden.

Der jüngere Lößabschnitt B 1 beginnt mit einer geringmächtigen Schicht verschleppter Gerölle (B 2). Er hat eine deutlich gröbere Körnung als der liegende Löß und wurde durch einen stärkeren Wind sedimentiert. Das primäre Aufwehen dieses Lösses, der im Liegenden des postglazialen Bodens schwache Verlehmungsspuren aufweist, ist durch das charakteristische Gefüge und das niedrige Volumengewicht genügend belegt.

Die postglaziale Pararendsina A. (= „flachgründiger Tschernosem“) ist im Oberboden durch Ackerbau gestört. Der im ursprünglichen Zustande erhaltene Unterboden steht den Böden des D-Komplexes, besonders dem Boden D 3, sehr nahe. In der Schicht A 3 gibt es aber vereinzelte Krotowinen, und das schwach entkalkte Liegende des Bodens (A 4) zeigt ein sekundär gestörtes Gefüge und ziemlich zahlreiche Wurmgänge. Diese Gänge reichen bis in die Tiefe von $2 \mathrm{~m}$ unter der Oberfläche.

Der Detritus in der Fraktion 0,1-0,2 der Probe L 1 (Ackerkrume) hat folgende Zusammensetzung: Quarz 70\%, Feldspat 12\%, Kalkstein 4\%, Biotit 2\%, Muskovit 1\%, I.imonit 5\%, Akzessorien 6\% Foraminiferen und Spongiennadeln fehlen.

Als Seltenheit wurden in dieser Fraktion glashelle autochthone Kalzit-Rhomboeder festgestellt, die für die holozänen Sedimente charakteristisch sind (vgl. LožEK-SEKYRAKuKLA-FeJFAR 1957).

Wenn wir alle unsere Untersuchungen kurz zusammenfassen, erhalten wir folgendes Ergebnis: das studierte Profil besteht aus 6 selbständigen, zeitlich aufeinander folgenden Lößdecken mit 9 autochthonen Böden (einschließlich der postglazialen Bodenbildung), von denen 7 einen humosen A-Horizont besitzen. Der untere und mittlere Profilabschnitt, der auch die fossilen Böden einschließt, besteht aus mergelartigen Erden mit relativ hohem $\mathrm{CaCO}_{3}-$ Gehalt (20-40\%). Deshalb sind alle fossilen Böden als Rendsinen entwickelt. 


\section{Die zeitliche Einordnung des Profils}

Bei der Altersbestimmung der einzelnen Schichten des untersuchten Profils muß man vor allem seine morphologische Lage in Betracht ziehen. Aus der Seehöhe der Profilsohle ergibt sich, daß der Lößkomplex jünger ist als die oberen Schichten der Terrasse IIIb, deren Oberkante in der Umgebung von Leitmeritz etwa in der Seehöhe von $175 \mathrm{~m}$ verläuft. Die erwähnte Terrassenstufe wird im Sinne des in Böhmen üblicherweise benützten Systems mit dem Altriß, also mit der Saale-Vereisung, parallelisiert. Abgesehen von der wenig wahrscheinlichen Möglichkeit jungpleistozäner Krustenbewegungen, deren Spuren bisher nirgends in der Umgebung von Leitmeritz beobachtet wurden, kann das untersuchte Profil höchstens rißeiszeit lich sein.

Im Sinne dieser Betrachtung halten wir den basalen Löß (G) für rißeiszeitlich, wobei das Jungriß, also die Kaltzeit, die der Warthe-Vereisung entspricht, an erster Stelle in Betracht kommt. Beide bodenbildenden Prozesse, welche die Oberfläche dieses Lösses betroffen haben, und zwar der ältere, der zur unvollkommenen Entkalkung, nicht aber zur Entstehung eines Humushorizontes führte, und der jüngere, dessen Produkt die älteste Mullrendsina ist, verliefen im Letzten Interglazial (=Eem, R/W). Dieser Bodenkompiex hat nämlich den Jungriß-Löß im Liegenden und eine Zone der Kryoturbation im Hangenden, durch welche die obere Rendsina (F 3) deformiert wurde. Diese kennzeichnet den Beginn des folgenden Glazials.

Im Hangenden der Rendsina F 3 liegt ein Schichtenpaket, dessen untere Glieder offensichtlich durch Erdfließen sedimentiert wurden (F 2), während die höheren Schichten durch Abspülung entstanden sind. Diese Abspülungssedimente gehen dann oszillierend über in einen Löß, auf dem sich eine ausgeprägte Rendsina (D 3) entwickelt hat, die das älteste Glied des oberen fossilen Bodenkomplexes darstellt, in dem sich eine beträchtliche Anderung der Klimaverhältnisse abspiegelt.

Die Ablagerung, die sich zwischen den fossilen Böden F 3 und D 3 befindet und deren obere Schichten durch Lößbildung gekennzeichnet sind, gliedern wir in das FrühwürmStadial ein (W 1 in den älteren Arbeiten), wobei die Protorendsina F 1 einer der warmen Schwankungen in diesem Zeitabschnitt entsprechen dürfte (vgl. Gross 1958, S. 164-166, Tab. S. 182). Der für ein Stadial verhältnismäßig feuchte Klimacharakter, der durch die Art der Sedimentation gekennzeichnet und auch faunistisch belegt ist, stimmt im allgemeinen mit den Beobachtungen in anderen Gebieten Mitteleuropas überein (BRUNNACKER 1957, 1958; FINK 1954, 1956; LožEK 1955).

Der Komplex der beiden mächtigen Humusböden D 1 und D 3 ist durch eine Zwischenschicht getrennt, die keine sicheren Spuren von Froststörungen auf weist. Beide Böden entstanden im ungefähr gleichen trockenen winterkalten Klima, wie es die aus wenig anspruchsvollen Arten zusammengesetzte Steppenfauna belegt. Daraus kann man schließen, daß sie das Produkt einer Wärmeschwankung sind, die sich klimatisch vom Interglazial scharf unterscheidet und deshalb als I $\mathrm{n}$ e $\mathrm{r} s \mathrm{t}$ a $\mathrm{d}$ i a 1 gewertet werden muß. In Hinsicht auf seine stratigraphische Position stellen wir diesen Schichtenkomplex in das Göttweiger Interstadial im Sinne von H. Gross (1956, 1958).

Der Komplex $\mathrm{C}$ besteht aus zwei flachgründigen Böden, die sich auf Löß und Abspülungsablagerungen entwickelt haben. Die basalen Schichten dieses Komplexes weisen deutliche Fließerscheinungen auf, welche für eine relativ kalte Klimaschwankung zeugen. Aus diesem Grunde stellen wir den Komplex C samt dem hangenden Komplex B bereits in das Hauptwürm (W 2 und 3 in den älteren tschechoslowakischen Arbeiten). Fraglich bleibt die Position des Paudorfer Interstadials in dieser Schichtenfolge und die Beziehungen der fossilen Böden im Abschnitt C zum Göttweiger Interstadial. Auf Grund des Materials, das in Leitmeritz zur Verfügung steht, kann man diese Frage nicht eindeutig beantworten. Wir sind aber der Meinung, daß die Böden C 3 und C 5 dem Göttweiger Interstadial zeitlich näher stehen und deshalb als Produkt kleiner Oszillationen am Anfang des Haupt- 
würms zu werten sind. Der Boden C 1 kann der Paudorfer Bodenbildung äquivalent sein; es ist aber nicht ausgeschlossen, daß er doch noch zu den erwähnten Anfangsoszillationen gehört und daß der dem Paudorfer Interstadial entsprechende Boden durch mächtige Solifluktion abgetragen wurde. Die erste Alternative betrachten wir als wahrscheinlicher, und zwar darum, weil der Boden C 1 an der Grenze zweier Lößlagen verschiedener Körnung liegt. Der erwähnte Boden beendet die Sedimentation des feinkörnigen Lösses C 2, während in seinem Hangenden eine neue grobkörnige Lößbildung beginnt, welcher erst höher eine Fließzone eingeschaltet ist.

Die Lösse des Komplexes B schließen außer der mächtigen unteren Fließzone noch zwei geringmächtige Solifluktionslagen ein, was an Verhältnisse erinnert, die aus anderen Gebieten der Tschechoslowakei von B. KLímA (1957) und K. ŽeberA (1958) beschrieben wurden. Der grobe Löß im oberen Abschnitt dieser Schichtenfolge hat auch bekannte Analogien in der Tschechoslowakei (vgl. z. B. die bisher unveröffentlichten Feststellungen von Fr. PвоŠEк in der Dzeravá-skala-Höhle in den Kleinen Karpathen).

Eine erweislich holozäne Sedimentation wurde nicht festgestellt; im Postglazial hat sich aber ein Boden von Pararendsina Typus („flachgründiger T'schernosem“) entwickelt.

\section{Vergleich mit anderen Profilen}

Aus dem Vergleich des Leitmeritzer Profils mit nahen jungpleistozänen Fundstellen ergibt sich eine auffallende Übereinstimmung mit der Schichtenfolge im unteren Arbeitsniveau der Ziegelei von Sedlec bei Prag, wo im Hangenden der Jungriß-Terrassen auf einem Jungriß-Löß ein fossiler Bodenkomplex liegt, der aus einer basalen braunen Verlehmungszone und einem Humushorizont besteht, die voneinander durch eine lichtere

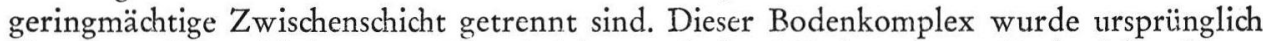
als „degradierter Tschernosem“ beschrieben und als „III. begrabener Boden“ bezeichnet (vgl. ProšEK \& LožEK 1957). Der Humushorizont ist von humosen kalkhaltigen Bodensedimenten mit fragmentarisch erhaltener hochinterglazialer Molluskenfauna überlagert, welche samt der stratigraphischen Position dieses Komplexes belegt, daß der als III. begrabener Boden bezeichnete Bodenkomplex dem Letzten Interglazial entspricht. In seinem Hangenden liegt eine Schichtenfolge von Lössen und lößartigen Ablagerungen mit einer geringmächtigen Humuseinlagerung und dann noch ein mächtiger Bodenkomplex, der aus zwei ausgeprägten Humushorizonten besteht, von denen der untere an der Basis einen braunen Verlehmungssaum aufweist. Dieser als „II. begrabener Boden “ bezeichnete Bodenkomplex wurde in Hinsicht auf seine stratigraphische Position im Profil mit dem Göttweiger Interstadial parallelisiert. Analoge, durch Funde hochinterglazialer Schnekkenfauna belegte Profile sind auch aus Letky und Jenerálka bekannt.

Alle erwähnten Schichtenfolgen entsprechen dem klassischen Profil in Stillfried an der March (FINK 1954, 1956). Dem öfters diskutierten fossilen Bodenkomplex Stillfried A entsprechen beide erwähnte Bodenkomplexe (d. h. der II. und III. begrabene Boden) in Sedlec, Letky und Jenerálka und die Schichtenfolge C, D und F in Leitmeritz. Für die innerböhmischen Verhältnisse ist kennzeichnend, daß die Sedimente, die den unteren und oberen Bodenkomplex voneinander trennen, im Vergleich mit Österreich und Südmähren relativ mächtig entwickelt sind, so daß sie immer als selbständige Lößdecke des Frühwürms („W 1“) beschrieben wurden. Nur in Jenerálka ist diese Lage so geringmächtig, daß die Übereinstimmung mit Stillfried praktisch vollkommen ist. Daraus ergibt sich, daß der Stillfrieder Komplex A sowohl das Göttweiger Interstadial als auch das Letzte Interglazial umfassen muß. Da allerdings die Parallelisierung dieser voneinander entfernten Profile sich noch nicht auf stratigraphische und petrographische Untersuchungen stützt, die mit erforderlicher Genauigkeit durchgeführt werden müßten, kann diese Feststellung bisher nicht als endgültig angesehen werden. 


\section{Die Position des letzten echten Interglazials in den Lößkomplexen}

Die Lösung des Problems der stratigraphischen Position des sicher belegten Letzten Interglazials in den Lößprofilen ist zur Kardinalfrage der Lößstratigraphie geworden. In der letzten Zeit zieht dieses Problem immer größere Aufmerksamkeit auf sich, denn seine endgültige Klärung wäre für den Fortschritt der Quartärstratigraphie von höchster Bedeutung. Zur Aufklärung der prinzipiellen Fragen hat die Studie von K. BRunNacker (1958) sehr beträchtlich beigetragen, die die Hauptansichten gegenüberstellt und einige wichtige Begriffe hervorhebt, deren Inhalt man klar bestimmen und definieren muß vor allem den Unterschied zwischen den Begriffen Interstadial und Interglazial und eine Klassifikation der quartären Klimaschwankungen überhaupt. Bei der Lösung dieser Fragen besteht ein großes Hindernis in der faziell abweichenden Entwicklung der fossilen Bodenkomplexe in verschiedenen klimatischen Regionen.

Unsere Studie hat sich zum Ziel gesetzt, zur Erklärung des Unterschiedes zwischen Interglazial und Interstadial beizutragen, der durch die Soengelsche (1925) Auffassung der sogenannten Vollgliederung des Quartärs erheblich verwischt wurde, was später zu einem großen Wirrwarr in der Stratigraphie und zur gegenseitigen Verwechslung klimatischer Schwankungen verschiedener Ordnung führte.

Wenn wir eine genaue Kennzeichnung der quartären Klimaschwankungen, besonders der warmen, festsetzen wollen, müssen wir uns auf ihre paläontologische Charakteristik stützen, die bisher als verläßlichstes Kriterium erscheint. Im Nordischen Vereisungsgebiet entspricht dem letzten echten Interglazial zweifellos die Eem-Warmzeit, welche durch die Vegetationsentwicklung, die aus vielen eingehend bearbeiteten Profilen bekannt ist, und durch die betreffende Meerestransgression genügend gekennzeichnet ist. Es ist allerdings ziemlich schwierig, bestimmte Horizonte der Lößschichtenfolgen direkt mit den Eem-Ablagerungen zu parallelisieren, die faziell völlig verschieden sind. Aus der Entwicklung der Eem-Flora ergibt sich aber mit Sicherheit, daß der hochinterglaziale Zeitabschnitt durch ein wärmeres und offenbar auch feuchteres Klima als in der Gegenwart gekennzeichnet war. Man kann also voraussetzen, daß die Fauna und Flora der der EemWarmzeit entsprechenden Periode im mitteleuropäischen Raume mindestens so wärmeund feuchtigkeitsliebend gewesen sein muß wie im postglazialen Klimaoptimum. Auch ihr Artenreichtum muß entsprechend groß sein, d. h. den heutigen Stand erreichen oder eher übertreffen.

In Mitteldeutschland gibt es eine Reihe von Travertinprofilen (Weimar, Taubach, Ehringsdorf, Burgtonna, Bilzingsleben, Osterode), die reiche Floren und Faunen sowie archäologische Funde geliefert haben und auf Grund aller brauchbaren stratigraphischen Kriterien dem letzten echten Interglazial des nordischen Raumes, d. h. der Eem-Warmzeit, entsprechen (vgl. WoldstedT 1950). Aus der Analyse des paläontologischen Inhaltes dieser Fundstellen ist ersichtlich, daß der letzten pleistozänen wärmeliebenden Flora die letzte Säugetierfauna mit Palaeoloxodon antiquus $\mathrm{F}_{\text {ALC. }}$ und Dicerorbinus mercki JäG. und die feuchtwarme Molluskenfauna mit der Leitart Helicigona banatica (Rossm.) entsprechen. Die Banatica-Fauna, welche klimatisch anspruchsvoller und artenreicher als die Molluskenbestände des postglazialen Klimaoptimums ist, muß als Aequivalent der AntiquusFauna betrachtet werden. Sie unterscheidet die letzte echte Warmzeit genügend von allen jüngeren Zeitabschnitten einschließlich des Postglazials.

Das Hauptziel der Lößpaläontologie ist jetzt, charakteristische warmzeitliche Fossilien in den Lößkomplexen zu entdecken, was allerdings auf große Schwierigkeiten stößt, $\mathrm{da}$ diese Ablagerungen bei weitem nicht so günstige Fossilisationsbedingungen bieten wie die Travertine oder limnischen Sedimente. Am günstigsten erscheinen die Möglichkeiten im Falle der Mollusken, dic im Löß unvergleichlich häufiger als die Knochenreste von Säugetieren vorkommen. Es ist gelungen, die dem Eem-Interglazial entsprechenden Banatica- Faunen in den Lößprofilen im Moldau-Tal nördlich von Prag festzustellen (Sedlec, 
Letky, Jenerálka), und zwar - wie bereits erwähnt - im Bereiche des „III. begrabenen Bodens“, der also letztinterglazial sein muß. Der im hangenden Lößkomplex auftretende fossile Bodenkomplex („II. begrabener Boden“) muß also eine warme Schwankung im Letzten Glazial (Würm) darstellen, d. h. das Interstadial W 1/2 der tschechoslowakischen Autoren, welches mit dem Göttweiger Interstadial identisch ist. Für diese Auffassung zeugen auch die Profile im Waagtal, besonders Zamarovce, wo allerdings die fossilen Böden in einer abweichenden Fazies entwickelt sind. Die Verhältnisse, die in Böhmen festgestellt wurden, scheinen also die Tatsache zu beweisen, daß im Bereich des Stillfrieder Komplexes A sowohl das Letzte Interglazial, als auch das Göttweiger Interstadial verborgen sind, deren fossile Bodenkomplexe nur lokal durch mächtigere Schichten von Lößmaterial voneinander getrennt sind. Dadurch wïrde a u ch der in der Einleitung erwähnte Widerspruch in der stratigraphischen Gliederung der Würmeiszeit gelöst.

Bei regionalen Parallelisierungen der Lößkomplexe muß man allerdings stets sehr vorsichtig sein, weil das Alter nur einer verhältnismäßig geringen Zahl solcher Profile tatsächlich als gesichert gelten kann. Es besteht ein berechtigter Verdacht, daß unter den sogenannten Würm-Profilen bis jetzt auch viele ältere Profile angeführt werden, deren obere Abschnitte abgetragen wurden, so daß die älteren Schichten bis zur Oberfläche reichen. Als Beispiel kann man das wohlbekannte Profil im oberen Arbeitsniveau der Ziegelei von Sedlec bei Prag erwähnen, das mehrmals als typisches Würm-Profil veröffentlicht wurde (vgl. z. B. R. LaIs 1951, S. 151, 143-145, Taf. 5-7). Die genaue Durchmessung der gesamten Lößwand zeigte aber klar, daß diese Schichtenfolge größtenteils $\mathrm{r}$ i $ß$ e is z e it li ch ist und daß die als Göttweiger Verlehmungszone beschriebene Bodenbildung in ein Interriß-Interstadial fällt. Aus demselben Grunde müssen auch alle hier veröffentlichten Folgerungen mit einer bestimmten Reserve betrachtet werden, solange man nicht eine absolute Datierung mit Hilfe von $\mathrm{C}^{14}$ durchführen kann.

In der Analyse der fossilen Molluskengesellschaften haben wir gezeigt, wie auffallend sich die Fauna des oberen Bodenkomplexes (MF 4, 3 und 2) von den letztinterglazialen Banatica-Faunen unterscheidet. Aus diesem Unterschied ergibt sich auch die genaue Bestimmung der Begriffe Interglazial und Interstadial, die man am besten durch den Vergleich beider Arten dieser Schwankungen mit den postglazialen Verhältnissen erklären kann. Das Interglazial gleicht in der Temperatur und Feuchtigkeit dem postglazialen Klima, bzw. kann es in seinem Höhepunkt noch übertreffen. Die Entwicklung der Fauna und Flora erinnert stark an die postglazialen Floren- und Faunenfolgen; allerdings erscheinen im Hochinterglazial in Mitteleuropa einige äußerst wärmebedürftige Arten, die im Postglazial nicht mehr in unsere Länder vorgedrungen sind, bzw. einige charakteristische ausgestorbene Säugetiere. Für das Interglazial ist weiter eine allgemeine Bewaldung sehr kennzeichnend, woraus man schließen kann, daß die Feuchtigkeit einen höheren Grad als im Postglazial erreichte. Demgegenüber ist das Interstadial durch eine verhältnismäßig arme Faura charakterisiert, welche für ein rauhes Festlandsklima und ein vorwiegend steppenartiges Gepräge der Landschaft zeugt. Feuchtigkeit und Temperatur erreichen bei weitem jicht die postglaziale Intensität, bleiben aber deutlich höher als in den Kaltzeiten. Die Lößbildung ist auf lange Zeit unterbrochen, und auf den vorhandenen Lößdecken bilden sich Steppenböden von Tschernosem-Charakter, die hier und da schwach degradiert sein können. Die auffallende dunkle Farbe dieser Bodenbildungen führte die Quartïrstratigraphen oft zur Überzeugung, daß es sich um ein Produkt des interglazialen Klimas handle, was aber nicht begründet ist, wenn man die geographische Verbreitung der Tschernoseme in Osteuropa und Nordasien in Betracht zieht. An der Nordgrenze ihrer Verbreitung treten diese Böden auch in recht rauhen Klimagebieten auf, die durch ein 
häufiges Vorkommen von solchen 'Tierarten gekennzeichnet sind, die in Mitteleuropa als typische Vertreter der kaltzeitlichen Fauna gelten. Deshalb ist auch die interstadiale Säugetierfauna nicht auffallend ausgeprägt. Aus den erwähnten Tatsachen ist klar ersichtlich, daß man die Begriffe Interglazial und Interstadial immer scharf auseinanderhalten muß, denn es handelt sich um Schwankungen verschiedener Ordnung (vgl. auch Brunnacker 1958), deren Unterschied man an folgendem Beispiel zeigen kann: Wäh rend das Klima des mitteleuropäischen Hochinterglazials annähernd den heutigen Klimaverhältnissen im Nordteilder Balkanhalbinsel entsprach, könnte man das interstadiale Klima mit den heutigen Klimabedingungen am Fuße des südlichen Uralsvergleichen.

Die beschriebenen klimatischen und faunistischen Züge gelten aber im Verlaufe der Würmeiszeit nur für das Göttweiger Interstadial, während die übrigen Schwankungen, z. B. das Paudorfer oder Alleröd-Interstadial, als viel schwächer e r s cheinen und Klimabedingungen aufweisen, die den kaltzeitlichen Verhältnissen noch näher stehen. Vom Standpunkte der Malakozoologie wurden diese Unterschiede bereits in den Aufsätzen über die Lößprofile des Waagtales genügend diskutiert (AMBrož-LožEK-ProšEK 1952; Prošek \& Ložek 1954, 1955), wo festgestellt wurde, daß die Molluskenfauna des Paudorfer Interstadials im allgemeinen kalt bleibt, aber im Vergleich mit der typischen Lößfauna ein deutlich feuchteres, man kann sagen „ozeanisches“ Gepräge hat. Mit dieser Beobachtung stehen auch die relativ schwach entwickelten Böden dieser Interstadiale im Einklang, die in den Lößprofilen immer vie l we n ige r a uffalle nd e r s c he in en als die Bodenbildungen des Göttweiger Interstadials oder des Letzten Interglazials. Die Interstadiale vom Paudorf- oder AllerödTypus stellen also noch eine niedrigere Ordnung von Schwankungen dar als das Göttweiger Interstadial, und in Ubereinstimmung mit dem Vorschlag von H. Movius wäre in ihrem Falle der Ausdruck "Oszillation" weit zutreffender als die Bezeichnung „Interstadial".

\section{Schluß}

1. In Leitmeritz (Nordböhmen) wurde eine Lößschichtenfolge eingehend stratigraphisch bearbeitet, die einen reich gegliederten jungpleistozänen Bodenkomplex einschließt.

2. Durch die petrographische Analyse, bei der verschiedene Miethoden einschließlich der Dünnschliffuntersuchung benützt wurden, wurden im Profil 6 Lößdecken und 8 autochthone fossile Bodenbildungen unterschieden, abgesehen von Schichten, die durch Erdfließen und Abspülung entstanden sind.

3. Im mittleren Abschnitt der untersuchten Schichtenfolge wurden Lösse mit beträchtlichem Anteil von Mergeldetritus festgestellt, der aus turonischen Sedimenten ausgeweht wurde. Diese Ablagerungen weisen ein typisches mikroskopisches Bild der Lösse auf, aber beim Schlämmen für die Kornanalyse erscheinen sie größtenteils als schluffige Tone. Ihr Kalkgehalt erreicht lokal fast $40 \%$. Die Böden, die sich auf diesem Untergrund entwickelt haben, gehören in die Rendsina-Gruppe und dürfen als Fazies der Tschernoseme betrachtet werden, die auf weniger kalkhaltigem Ausgangsmaterial entstanden sind.

4. Im Profil kann man zwei Hauptkomplexe fossiler Böden unterscheiden, die voneinander durch eine Lößlage getrennt sind. Der jüngste und der älteste Boden entbehren eines deutlichen Humushorizontes. Zwischen ihnen sind 3 mächtigere Rendsinen und 3 flachgründige Humusbildungen eingeschlossen.

5. In den Bodenkomplexen wurde eine Molluskenfauna festgestellt, die aus klimatisch wenig anspruchsvollen Steppenarten besteht. In beiden Hauptrendsinen des oberen Boden- 
komplexes tritt eine ausgeprägte Steppenfauna auf, die beweist, daß diese Böden in einem verhältnismäßig rauhen und trockenen Klima entstanden sind, das sowohl vom interglazialen als auch vom kaltzeitlichen Klima scharf verschieden war. In den Bodensedimenten auf der Oberfläche des unteren Bodenkomplexes kommt eine Steppenfauna mit beträchtlicher Beimischung von Waldsteppenarten vor, die den Klimabedingungen an der Grenze einer Warmzeit und der folgenden Kaltzeit entspricht. In den eigentlichen Böden des unteren Komplexes wurden keine Konchylien festgestellt.

6. Das untersuchte Profil muß in Hinsicht auf die Höhenbeziehungen zu den Elbeterrassen als post-altriß-eiszeitlich betrachtet werden. Der untere Bodenkomplex entspricht dem Letzten Interglazial, beide humose Haupthorizonte des oberen Komplexes dem Göttweiger Interstadial, während man die hangenden schwachen Bodenbildungen bereits in die Anfangsphase des Hauptwürms stellen kann.

7. Der Leitmeritzer Aufschluß zeigt eine große Übereinstimmung mit den Profilen von Sedlec bei Prag, Jenerálka und Letky, wo das Letzte Interglazial durch die hochinterglaziale Schneckenfauna verläßlich belegt ist. Alle diese Profile stimmen mit dem klassischen Aufschluß von Stillfried überein und weisen darauf hin, das der in der Literatur oft diskutierte Komplex Stillfried A sowohl das Letzte Interglazial als das Göttweiger Interstadial umfaßt. Diese Feststellung muß allerdings durch eingehende, mit modernen Methoden durchgeführte stratigraphische Untersuchungen an allen erwähnten Orten bestätigt werden.

8. Der Versuch, die jungpleistozänen Warmphasen zu charakterisieren, führte zur Feststellung scharfer Unterschiede zwischen dem Letzten Interglazial und dem Göttweiger Interstadial. Es erscheint allerdings zweckmäßig, auch dieses Interstadial als Schwankung höherer Ordnung von den übrigen letztglazialen Warmphasen zu unterscheiden, für welche der Termin „Oszillation" in Ubereinstimmung mit H. Movius geeigneter wäre.

\section{Schriftum :}

Ambrož, V., LožEK, V. \& ProšEK, Fr.: Pléistocène récent aux environs de Moravany près Piešt'any sur le Váh (Slovaquie occidentale). - Anthropozoikum 1 (1951), S. 53-142, Taf. I-IV, Praha 1952.

Bárta, J.: Pleistocénne piesočné duny pri Seredi a ich paleolitické a mezolitické osídlenie (Pleistozäne Sanddünen bei Sered' und ihre paläolithische und mesolithische Besiedlung). Slovenská Archeológia 5, S. 5-72, Bratislava 1957.

BRAnDtner, Fr.: Über die relative Chronologie des jüngeren Pleistozins Niederösterreichs. Archeologia Austriaca 5, S. 101-113, Wien 1950. - - Jungpleistozäner Löß und fossile Böden in Niederösterreich. - Eiszeitalter und Gegenwart 4/5, S. 49-82, Öhringen 1954. - - Lößstratigraphie und paläolithische Kulturabfolge in Niederösterreich und in den angrenzenden Gebieten. - Ebendort 7, S. 127-175, Öhringen 1956.

BrunnackeR, K.: Würmeiszeitlicher Löß und fossile Böden in Mainfranken. - Geologica Bavarica 25, S. 22-38, München 1955. - - Die Geschichte der Böden im jüngeren Pleistozän in Bayern. - Geologica Bavarica 34, S. 1-95, Taf. I-II, München 1957. - - Zur Parallelisierung des Jungpleistozäns in den Periglazialgebieten Bayerns und seiner östlichen Nachbarländer. - Festschrift zum 70. Geburtstag von Paul WoldstedT; Geologisches Jahrbuch 76, S. 129-149, Hannover 1958.

FINK, J.: Die fossilen Böden im österreichischen Löß. - Quartär 6, S. 85-108, Bonn 1954. - - Zur Korrelation der Terrassen und Lösse in Österreich. - Eiszeitalter und Gegenwart 7, S. 49-77, OOhringen 1956.

Freising, H.: Neue Ergebnisse der Lößforschung in Württemberg, - Jahreshefte geol. Abt. württemberg. statist. Landesamt 1, S. 54-59, Stuttgare 1951. - Gliederung und Alter des deutschen Lösses (Zusammenfassung). - Actes du IV Congrès International du Quaternaire (Rome-Pise, Août-Septembre 1953) I, S. 340, Roma 1956.

Götzinger, G.: Zur Gliederung des Lösses. Leimen- und Humuszonen im Viertel unter dem Manhartsberge. - Verhandl. geol. Bundesanstalt 1935, S. 126-132, Wien 1935.

Gross, H.: Das Göttweiger Interstadial, ein zweiter Leithorizont der letzten Vereisung. - Eiszeitalter und Gegenwart 7, S. 87-101, Ohringen 1956. - - Die bisherigen Ergebnisse von 
C14-Messungen und paläontologischen Untersuchungen für die Gliederung und Chronologie des Jungpleistozäns in Mitteleuropa und den Nachbargebieten. - Ebendort 9, S. 155-187, OOhringen 1958.

KLímA, B.: UUbersicht über die jüngsten paläolithischen Forschungen in Mähren. - Quartär 9, S. 85-130, Taf. IV-X, Bonn 1957.

KubiëNA, W. L.: Zur Mikromorphologie, Systematik und Entwicklung der rezenten und fossilen Lößböden. - Eiszeitalter und Gegenwart 7, S. 1C2-112, Taf. II-V, Öhringen 1956.

I.AIs, R.: Über den jüngeren Löß in Niederösterreich, Mähren und Böhmen. - Ber. naturf. Ges. Freiburg i. Br. 41, S. 119-168, Taf. 1-10, Freiburg 1951. - - Ein Lößprofil bei Moravany in Mähren. - Ebendort 48, S. 91-118, Freiburg 1958.

Licharev, I. M. \& Rammelmeyer, E. S.: Nazemnyje Molljuski fauny SSSR. - S. 1-511, MoskvaLeningrad 1952.

LožEK, V.: Zpráva o výzkumu kvarterních měkkýšů v roce 1952. - Anthropozoikum 3 (1953), S. 129-134, Praha 1954(a). - - Zpráva o paleontologickém výzkumu cihelny v Zájezdê u Buštěhradu. - Ebendort 3 (1953), S. 135-138, Praha 1954 (b). - - Měkkýši československého kvartéru (Mollusken des tschechoslowakischen Quartärs). - Rozprav y Ústředního ústavu geologického 17, S. 1-510, 3 Beil., Taf. I-XII, Praha 1955. - - Výzkum ložisek přirozených hnojiv $v$ oblasti Českého masivu. - Zprávy o geologických výzkumech v roce 1957, S. 123-124, Praha 1959.

Ložek, V., Sekyra, J., Kukla, J. \& FejFar, O.: Výzkum Velké Jasovské jeskyně (Die Durchforschung der Großen Jasover Höhle). - Anthropozoikum 6 (1956), S. 193-282, Taf. I-X, Praha 1957.

Movius, H. L.: Radiocarbon Dates and Palaeolithic Archaeology in Central and Western Europe. - Actes du V Congrès International du Quaternaire, Madrid-Barcelone 1957.

Müller-Beck, Hj.: Paläolithische Kulturen und pleistozäne Stratigraphie in Süddeutschland. Eiszeitalter und Gegenwart 8, S. 116-140, Ohringen 1957.

Prošex, Fr.: Le Szeletien en Slovaquie. - Slovenská Archeologia 1, S. 133-194, Taf. I-XII, Bratislava 1953.

Prošex, Fr. \& LožEK, V.: Sprašový profil v Bance u Piešt’an (západní Slovensko) (The Loess Section at Banka near Piešt'any (Western Slovakia). - Anthropozoikum 3 (1953), S. 301-323, Taf. I-II, Praha 1954. - - Výzkum sprašového profilu v Zamarovcích u Trenč́na (Untersuchung des Lößprofiles von Zamarovce bei Trenčín). - Ebendort 4, (1954), S. 181-211, Taf. I-II, Praha 1955. - - Stratigraphische Übersicht des tschechoslowakischen Quartärs. - Eiszeitalter und Gegenwart 8, S. 37-90, Öhringen 1957.

Sozrgel, W.: Die Gliederung und absolute Zeitrechnung des Eiszeitalters. - Fortschritte der Geologie und Paläontologie 13, S. I-VI, 125-251, 3 Taf., Berlin 1925.

VÉRTES, L.: Les conditions de l'interstadial Würmien I/II hongrois élucidées par l'examen des remplissages de grottes. - Acta geologica 3, S. 393-407, Budapest 1955.

VetTens, H. \& GötzINGER, H.: Exkursion in das Lößgebiet des niederösterreichischen Weinviertels und angrenzenden Waldviertels. - Führer für die Quartär-Exkursionen in Österreich, I. Teil, S. 35-39, Taf. 3-4, Wien 1936.

Weidendach, Fr.: Gedanken zur Lößfrage. - Eiszeitalter und Gegenwart 2, S. 25-36, Ơhringen 1952. - - Zeitliche Einordnung der jungpleistozänen Ablagerungen Mitteleuropas. - Actes du IV Congrès International du Quaternaire (Rome-Pise, Aoút-Septembre 1953) II, S. 891-897, Roma 1956.

Woldstedt, P.: Norddeutschland und angrenzende Gebiete im Eiszeitalter. - Geographische Handbücher, S. I-XII, 1-464, Tab. 1-12, Stuttgart 1950. - - Uber die Gliederung der Würm-Eiszeit und die Stellung der Lösse in ihr. - Eiszeitalter und Gegenwart 7, S. 78 bis 86, Ohringen 1956. - - Das Eiszeitalter, Grundlinien einer Geologie des Quartärs. Zweiter Band: Europa, Vorderasien und Nordafrika im Eiszeitalter, 2. Aufl., S. I-VII, 1-438, Taf. I, Stuttgart 1958.

ZaHÁLKA, Bř.: Terasy Vltavy a Labe mezi Veltrusy a Roudnicí n. L. (Les terrasses de la Vltava et de la Labe entre Veltrusy et Roudnice n. L.) - Sborník Státního geologického ústavu ČSR 13, S. 377-462, Taf. I-II, Praha 1946.

ZÁRuba, Q. \& Hromada, K.: Technicko-geologický rozbor území města Kutné Hory (GeologicoTechnical Surveying of the Town of Kutná Hora). - Geotechnica 9, S. 1-47, Taf. I bis III, 2 Beil., Praha 1950.

ŽEberA, K.: Československo ve starší době kamenné (Die Tschechoslowakei in der älteren Steinzeit). - S. 1-214, Taf. 1-88, Praha 1958.

Manuskr. eingeg. 6. 4. 1959.

Anschrift der Verf.: Dr. Vojen Ložek und Dr. Jiří Kukla, Geologische Zentralanstalt, Prag I, Malá Strana, Malostranské náměstí 18. 